\title{
PENALTY-FINITE ELEMENT METHODS FOR THE ANALYSIS OF STOKESIAN FLOWS*
}

\author{
J. Tinsley ODEN \\ TICOM and the Department of Aerospace Engineering and Engineering Mechanics, \\ University of Texas at Austin, Austin, TX 78712, USA \\ Noboru KIKUCHI \\ Department of Mechanical Engineering and Applied Mechanics, University of Michigan, Ann Arbor, \\ MI 48109, USA \\ Young Joon SONG \\ Korean Military Academy, Seoule, Korea
}

Received 20 October 1981

\begin{abstract}
A study of a class of finite element methods for the analysis of Stokes' problem based on the use of exterior penalty formulations is described. The effects of selective reduced integration (i.e., the use of quadrature rules for integrating the penalty terms which are of lower order than that required to integratc polynomial approximations of these terms exactly) are investigated. Error estimates are derived and the numerical stability of these methods, as depicted by a special Babuska-Brezzi condition, is explored in some detail. The results of several numerical experiments with these methods are also given.
\end{abstract}

\section{Introduction}

This study is concerned with the numerical analysis of a class of finite element methods for Stokesian flow problems of the type

$$
\begin{array}{ll}
\left.\begin{array}{rl}
-\mu \Delta u+\nabla p=f \\
\operatorname{div} u=0
\end{array}\right\} & \text { in } \Omega, \\
\boldsymbol{u}=\mathbf{0} & \text { on } \partial \Omega,
\end{array}
$$

where $\mu$ is the viscosity, $u$ the velocity field, $p$ the hydrostatic pressure, $f$ the applied body force density, and $\Omega$ is an open bounded domain in $\mathbb{R}^{n}$.

The methods under study here employ exterior penalty schemes to handle the incompressibility condition, div $\boldsymbol{u}=0$. Such methods have received considerable attention in the engineering literature in recent years. This popularity has been primarily because they appear to provide a device for eliminating the hydrostatic pressure $p$ from the formulation, thereby

${ }^{*}$ This work was completed during the course of a research program sponsored by the U.S. Air Force, Office of Scientific Research under Contract F-49620-78-C-0083. 
reducing dramatically the number of unknowns. If needed, proponents argue, the penalty approach allows one to obtain an approximation of $p$ a posteriori from the computed velocities with little computational effort.

Finite element methods based on such strategies have been proposed by several authors. We mention, in particular, the works of Fried [11], Malkus [21], Hughes [14, 15], Malkus and Hughes [22], Hughes, Taylor and Levy [16], Reddy [23] and Zienkiewicz, Taylor and Too [25]. These authors have determined, on the basis of numerical experiments, that it is necessary to use 'reduced integration' of the penalty terms in such formulations in order to obtain results which appear to be physically reasonable. By 'reduced integration' we mean the practice of using an approximate quadrature rule for integrating the penalty terms (such as $\left.\left(\operatorname{div} \boldsymbol{u}_{\varepsilon}, \operatorname{div} v\right)_{L^{2}(\Omega)}\right)$ in the formulation which is of lower order than that required to integrate these terms exactly. We discuss the use of reduced integration and its implications on the behavior of these methods in Section 4 of this paper. The equivalence of penalty methods of this type with certain mixed methods has been pointed out by Malkus and Hughes [22] and Bercovier [3]; also, the convergence of certain finite element methods based on penalty formulations of problems with linear equality constraints has been studied by Bercovier [3] and Bercovier and Engelman [4], but, unfortunately, under assumptions which do not hold for any of the methods of interest here.

The reduced-integration-penalty schemes considered here exhibit interesting and deceptive numerical stability characteristics. We show herein that the numerical stability of these methods is governed by a discrete inf-sup condition, referred to here as the LBB-condition after related work of Ladyszhenskaya [19], Babuska [1, 2], and Brezzi [5, 6], and given by equation (4.9). Generally speaking, those methods for which this condition holds with a stability parameter $\alpha_{h}$ independent of the mesh size $h$ are stable. If $\alpha_{h}$ is dependent on $h$, then the method may be unstable. In such cases if the exact solution is not very regular, erratic oscillations in the approximate solutions, particularly the pressures, are experienced.

In Section 6 of this paper, we develop examples of two popular elements for which we are able to show that $\alpha_{h}=\mathrm{O}\left(h^{2}\right)$. Yet, in special circumstances, particularly in the case of very smooth solutions on regular uniform meshes, these unstable methods can produce acceptable approximations of the velocities. In such cases, however, the pressure approximation is especially delicate and may diverge (in $L^{2}(\Omega)$ ) as the mesh is refined. A theory explaining this special behavior of the velocity approximation for smooth solutions has been advanced by Johnson and Pitkaranta [18] for a special element and uniform meshes on rectangular domains.

It is important to note that projection or 'filtering' schemes can be devised which allow one to modify the pressure approximations in such a way that a stability parameter $\alpha_{h}$ independent of $h$ can be produced. Such operations stabilize many of the otherwise unstable methods of this type and can lead to schemes which exhibit optimal rates-of-convergence for both the velocities and the pressures. We describe such a scheme in the present work in Section 8 . Results of a representative numerical experiment are discussed briefly in Section 9.

\section{Stokes' problem}

The classical model of Stokes of the steady, uniform flow of a viscous incompressible fluid can be characterized by the following variational boundary-value problem. 
Find $(u, p) \in V \times Q$ such that

$$
a(\boldsymbol{u}, \boldsymbol{v})-(p, \operatorname{div} v)=f(\boldsymbol{v}), \quad \forall v \in V, \quad(q, \operatorname{div} \boldsymbol{u})=0, \quad \forall q \in Q .
$$

Here $V$ is the space of admissible velocities, here given as the Sobolev space $V=\left(H_{0}^{1}(\Omega)\right)^{n}$ equipped with the norm,

$$
\|\boldsymbol{v}\|_{1}=\left\{\int_{\Omega} \sum_{i, j=1}^{n} \frac{\partial v_{i}}{\partial x_{j}} \frac{\partial v_{i}}{\partial x_{j}} \mathrm{~d} x\right\}^{1 / 2}
$$

with $\Omega$ an open bounded region in $\mathbb{R}^{n}$, and

$$
Q=\left\{q \in L^{2}(\Omega) \mid \int_{\Omega} q \mathrm{~d} x=0\right\}
$$

with the norm

$$
\|q\|_{0}=\left\{\int_{\Omega} q^{2} \mathrm{~d} x\right\}^{1 / 2}
$$

It follows from [18, p. 34] that the space $Q$ is equivalent to the quotient space of $L^{2}(\Omega)$ modulo elements in the closed subspace $\operatorname{ker} B^{*}$ where

$$
\operatorname{ker} B^{*}=\{q \in Q \mid(q, \operatorname{div} v)=0, \forall v \in V\}
$$

where $(\cdot, \cdot)$ denotes the $L^{2}(\Omega)$-inner product. Obviously, $B^{*}$ corresponds to the gradient in $\mathbb{R}^{n}$ plus boundary conditions, and in the present case ker $B^{*}=$ constant functions on $\Omega$ \}. In $(2.1), a(\cdot, \cdot)$ is the bilinear form on $V \times V$ given by

$$
a(\boldsymbol{u}, \boldsymbol{v})=\mu \int_{\Omega} \sum_{i, j=1}^{n} \frac{\partial u_{i}}{\partial x_{j}} \frac{\partial v_{i}}{\partial x_{j}} \mathrm{~d} x, \quad \forall \boldsymbol{u}, \boldsymbol{v} \in V,
$$

$\mu$ being the viscosity of the fluid, and $f$ is a bounded linear functional on $V$ given by

$$
f(v)=\int_{\Omega} f \cdot v \mathrm{~d} x, \quad \forall v \in V
$$

where $f \in\left(L^{2}(\Omega)\right)^{n}$ is the given body force.

Throughout this paper, we shall assume that

(1) $\mu$ is a given positive number,

(2) $f \in \boldsymbol{H}^{s-2}(\Omega) \equiv\left(H^{s-2}(\Omega)\right)^{n}, s \geqslant 2$,

(3) the domain $\Omega$ is Lipschitzian.

Under these conventions and assumptions, the following results hold. 
THEOREM 2.1. Let conditions (2.8) hold. Then (i) there exists a constant $\alpha>0$ such that

$$
\alpha\|q\|_{0} \leqslant \sup _{v \in V-\{0\}} \frac{(q, \operatorname{div} v)}{\|v\|_{1}}, \forall q \in Q
$$

and (ii) there exists a unique solution $(u, p) \in V \times Q$ to problem (2.1). If, in addition, $\Omega$ is of class $\mathscr{C}^{s}, s \geqslant 2$, then

$$
u \in\left(H^{s}(\Omega)\right)^{n} \cap V \quad \text { and } \quad p \in H^{s-1}(\Omega) .
$$

Proofs of results such as this, together with many additional details on this problem, can be found, for example, in the book of Temam [24]. Condition (2.9) was derived in a different but equivalent form by Ladyszhenskaya [19] and, in fact, holds under much weaker hypothesis than those stated in this theorem. Conditions similar to (2.9) have been developed by Babuska [1] and Brezzi [5] in the study of elliptic problems with constraints, and because of this history, we shall refer to (2.9) as the 'LBB-condition'.

\section{A penalty-formulation of the Stokes' problem}

The mixed variational problem (2.1) also characterizes the solution of a constrained minimization problem: minimize the energy

$$
J: V \rightarrow \mathbb{R} ; \quad J(v)=\frac{1}{2} a(v, v)-f(v),
$$

subject to the constraint that $\operatorname{div} v=0$. Thus, the hydrostatic pressure $p$ is a Lagrange multiplier associated with the incompressibility constraint $\operatorname{div} \boldsymbol{u}=0$. A direct finite-element approximation of problem (2.1) leads to so-called mixed finite element methods which have been studied extensively in the literature.

An alternative formulation to (2.1) is provided by the notion of exterior penalties whereby (2.1) is replaced by a family of perturbations consisting of unconstrained problems depending on a penalty parameter $\varepsilon>0$. A significant advantage in such penalty formulations is that the hydrostatic pressure $p$ does not appear explicitly in the formulation-thus suggesting that corresponding finite element schemes can be constructed which have significantly fewer unknowns than standard mixed methods.

Let $\varepsilon$ be an arbitrary positive number. Then a penalty approximation of the variational problem (2.1) consists of seeking $\boldsymbol{u}_{\varepsilon} \in V$ such that

$$
a\left(u_{\varepsilon}, v\right)+\varepsilon^{-1}\left(\operatorname{div} u_{\varepsilon}, \operatorname{div} v\right)=f(v), \quad \forall v \in V .
$$

It is easily shown that there exists a unique solution $\boldsymbol{u}_{\varepsilon}$ to (3.2) for each $\varepsilon>0$. It is also useful to note that this equation characterizes minimizers of the penalized energy functional,

$$
J_{\varepsilon}: J \rightarrow \mathbb{R} ; \quad J_{\varepsilon}(v)=\frac{1}{2} a(v, v)-f(v)+\frac{1}{2} \varepsilon^{-1}\|\operatorname{div} v\|_{0}^{2}
$$


over all of $V$, the term $\frac{1}{2} \varepsilon^{-1}\|\operatorname{div} v\|_{0}^{2}$ describing an exterior penalty associated with the constraint $\operatorname{div} v=0$.

To appreciate the relationship between (3.2) and (2.1), it is informative to note that the solution $(u, p) \in V \times Q$ of (2.1) is a saddle point of the functional $L: V \times Q \rightarrow \mathbb{R}$,

$$
L(v, q)=J(v)-(q, \operatorname{div} v)
$$

whereas saddle points $\left(u_{\varepsilon}, p_{\varepsilon}\right)$ of the perturbed Lagrangian $L_{\varepsilon}: V \times Q \rightarrow \mathbb{R}$,

$$
L_{\varepsilon}(v, q)=L(v, q)-\frac{1}{2} \varepsilon\left\|_{q}\right\|_{0}^{2}
$$

are characterized by the system

$$
\begin{aligned}
& a\left(u_{\varepsilon}, v\right)-\left(p_{\varepsilon}, \operatorname{div} v\right)=f(v), \quad \forall v \in V, \\
& -\left(q, \operatorname{div} u_{\varepsilon}\right)-\varepsilon\left(q, p_{\varepsilon}\right)=0, \quad \forall q \in Q .
\end{aligned}
$$

Since

$$
\int_{\Omega} \operatorname{div} \boldsymbol{u}_{\varepsilon} \mathrm{d} x=0
$$

we can eliminate the perturbed hydrostatic pressure from the last equation to obtain

$$
p_{\varepsilon}=-\frac{1}{\varepsilon} \operatorname{div} u_{\varepsilon} \quad \text { in } Q
$$

which, upon introduction into the first equation in (3.6) yields precisely the problem (3.2).

Thus, the exterior penalty formulation (3.2) arising from the minimization of the functional $J_{\varepsilon}$ in (3.3) is equivalent to the perturbed Lagrange formulation characterized by (3.6). Of special significance, however, is the observation that a penalty approximation $p_{\varepsilon}$ of the hydrostatic pressure can also be obtained via (3.7) oncc the solution $\boldsymbol{u}_{\varepsilon}$ to (3.2) has been determined. This again suggests that when a variational formulation such as (3.2) is used as a basis for constructing finite-element methods, schemes can be devised which produce results analogous to standard mixed methods but which lead to numerical methods which require considerably less computational effort. As we shall see, these apparent advantages are not always attainable.

We next establish the basic convergence theorem for the penalty method (3.2).

THEOREM 3.1. Let $u_{\varepsilon} \in V$ be the solution of (3.2) of given $\varepsilon>0$ and let $p_{\varepsilon}$ be given by (3.7). Then the sequence $\left\{\left(u_{\varepsilon}, p_{\varepsilon}\right)\right\}$ obtained from such solutions as $\varepsilon \rightarrow 0$ converges strongly $V \times Q$ to the solution $(u, p)$ of (2.1). Moreover, the estimate

$$
\left\|\boldsymbol{u}-\boldsymbol{u}_{\varepsilon}\right\|_{1}+\left\|p-p_{\varepsilon}\right\|_{0} \leqslant C \varepsilon
$$

holds with $C$ a constant independent of $\varepsilon$. In particular, 


$$
\left\|\boldsymbol{u}_{\varepsilon}-\boldsymbol{u}\right\|_{1} \leqslant \alpha^{-1}\|p\|_{0} \varepsilon \text { and }\left\|p-p_{\varepsilon}\right\| \leqslant 2 \mu \alpha^{-2}\|p\|_{0} \varepsilon
$$

where $\alpha$ is the constant in (2.9) and $\mu$ is the viscosity.

PROOF. It is sufficient to prove (3.9). Subtracting (2.1) from (3.6), yields

$$
a\left(\boldsymbol{u}_{\varepsilon}-\boldsymbol{u}, \boldsymbol{v}\right)=\left(p_{\varepsilon}-p, \operatorname{div} \boldsymbol{v}\right), \quad \forall \boldsymbol{v} \in V .
$$

An application of condition (2.9) leads to the relation,

$$
\alpha\left\|p_{\varepsilon}-p\right\|_{0} \leqslant 2 \mu\left\|\boldsymbol{u}_{\varepsilon}-\boldsymbol{u}\right\|_{1} \quad\left(p_{\varepsilon}-p \in Q\right) .
$$

Setting $v=\boldsymbol{u}_{\boldsymbol{\varepsilon}}-\boldsymbol{u}$ in (3.10) we obtain

$$
2 \mu\left\|\boldsymbol{u}_{\varepsilon}-\boldsymbol{u}\right\|_{1}^{2} \leqslant a\left(\boldsymbol{u}_{\varepsilon}-\boldsymbol{u}, \boldsymbol{u}_{\varepsilon}-\boldsymbol{u}\right)=\left(p_{\varepsilon}-p, \operatorname{div}\left(\boldsymbol{u}_{\varepsilon}-\boldsymbol{u}\right)\right) .
$$

Since $\operatorname{div} u=0$ in $L^{2}(\Omega),\left(p_{\varepsilon}-p, \operatorname{div}\left(\boldsymbol{u}_{\varepsilon}-u\right)\right)=\left(p_{\varepsilon}-p, \operatorname{div} \boldsymbol{u}_{\varepsilon}\right)$.

Because of the identification (3.7) we have $\left(p_{\varepsilon}-p,-\varepsilon p_{\varepsilon}\right) \leqslant\left(p_{\varepsilon}-p, p\right) \varepsilon$. Thus,

$$
2 \mu\left\|\boldsymbol{u}_{\varepsilon}-\boldsymbol{u}\right\|_{1}^{2} \leqslant\left\|p_{\varepsilon}-p\right\|_{0}\|p\|_{0} \varepsilon
$$

and combining (3.10) and (3.11) implies $\left\|\boldsymbol{u}_{\varepsilon}-\boldsymbol{u}\right\|_{1} \leqslant\|p\|_{0}(\varepsilon / \alpha)$. The second estimate in (3.9) now follows from (3.11).

Results similar to these have been obtained by much lengthier arguments by Bercovier [3], Reddy [23], and others. Generalizations of this proof for obtaining $\varepsilon$-convergence estimates to nonlinear boundary-value problems can be found in [17].

\section{Finite element approximations}

We now construct a family of finite-dimensional subspaces $\left\{V_{h}\right\}$ of the space $V$ using conforming finite elements on a suitable discretization $\Omega_{h}$ of $\Omega$, spanned by continuous, piecewise-polynomial basis functions. The index $h$ is, as usual, the mesh parameter. We will generally assume that the family $\left\{V_{h}\right\}$ is generated by regular refinements of the mesh and that $\Omega_{h}$ coincides with $\Omega$ ( $\Omega$ is, e.g., polygonal). More will be said about the approximation properties assumed for $V_{h}$ later.

In anticipation of some numerical difficulties to be addressed below, we will use numerical quadrature to evaluate certain integrals appearing in our approximation of the penalized problem. In particular, we shall evaluate the $L^{2}$-inner product of two functions $f, g \in C^{\circ}(\bar{\Omega})$ by means of numerical quadrature formulas $I(\cdot, \cdot)$ of the type

$$
\begin{aligned}
& I(f, g)=\sum_{e=1}^{E} I_{e}(f, g), \\
& I_{e}(f, g)=\sum_{i=1}^{G} W_{i}^{e} f\left(\xi_{i}^{e}\right) g\left(\xi_{i}^{e}\right), \quad W_{i}^{e} \geqslant 0 .
\end{aligned}
$$


Here $E$ is the total number of elements in the mesh, and $\left\{\xi_{i}^{e}, W_{i}^{e}\right\}$ is the set of quadrature points and weights within an element $\Omega_{e}, 1 \leqslant i \leqslant G$. Thus

$$
I(f, g) \approx \int_{\Omega} f g \mathrm{~d} x \text { and } I_{e}(f, g) \approx \int_{\Omega_{e}} f g \mathrm{~d} x .
$$

Our approximation of the penalized problem (3.2) then assumes the following form.

$$
\boldsymbol{u}_{\varepsilon}^{h} \in V_{h}: \quad a\left(\boldsymbol{u}_{\varepsilon}^{h} \boldsymbol{v}^{h}\right)+\varepsilon^{-1} I\left(\operatorname{div} \boldsymbol{u}_{\varepsilon}^{h}, \operatorname{div} \boldsymbol{v}^{h}\right)=f\left(\boldsymbol{v}^{h}\right), \quad \forall \boldsymbol{v}^{h} \in V_{h} .
$$

We first establish the solvability of (4.2).

THEOREM 4.1. Under the above conventions and the hypotheses of Theorem 2.1, there exists a unique solution $\boldsymbol{u}_{c}^{h}$ of (4.2) for each $h$ and $\varepsilon>0$. Moreover, for each fixed $h,\left\|u_{\varepsilon}^{h}\right\|_{1}$ is uniformly bounded in $\varepsilon$. Finally, there exists a constant $C$ independent of $\varepsilon$ such that

$$
I\left(\operatorname{div} \boldsymbol{u}_{\varepsilon}^{h}, \operatorname{div} \boldsymbol{u}_{\varepsilon}^{h}\right)=\sum_{e=1}^{E} \sum_{j=1}^{G} W_{j}^{e}\left(\operatorname{div} \boldsymbol{u}_{\varepsilon}^{h}\left(\xi_{j}^{e}\right)\right)^{2} \leqslant C \varepsilon
$$

PROOF. The existence of a unique solution $\boldsymbol{u}_{\varepsilon}^{h}$ to (4.2) and its uniform boundedness follow easily from the coerciveness of $a(\cdot, \cdot)$ and the fact that $V_{h} \subset V$. Estimate (4.3) follows from (4.2) upon setting $\boldsymbol{v}^{h}=\boldsymbol{u}_{\varepsilon}^{h}$ and recalling that $\left\|\boldsymbol{u}_{\varepsilon}^{h}\right\|_{1}$ is bounded independent of $\varepsilon$.

Notice that (4.3) indicates that the incompressibility condition is satisfied at the quadrature points $\xi_{j}^{e}$ in the limit as $\varepsilon$ tends to zero:

$$
\operatorname{div} u_{\varepsilon}^{h}\left(\xi_{j}^{e}\right) \rightarrow 0 \quad \text { as } \varepsilon \rightarrow 0 ; \quad 1 \leqslant j \leqslant G, 1 \leqslant e \leqslant E .
$$

The fact that (4.2) is uniquely solvablc for $\boldsymbol{u}_{\varepsilon}^{h}$ is, of course, no indication that (4.2) provides an acceptable approximation of (2.1). Since the functions $v^{h} \in V_{h}$ are polynomials, it is always possible to choose the order $G$ of the quadrature rule to be high enough that $I\left(\operatorname{div} \boldsymbol{u}_{\varepsilon}^{h}\right.$, div $\left.\boldsymbol{v}^{h}\right)$ yields the exact inner product $\left(\operatorname{div} \boldsymbol{u}_{\varepsilon}^{h}\right.$, div $\left.\boldsymbol{v}^{h}\right)$. However, for all practical purposes, the exact evaluation of these penalty terms does not produce a meaningful approximation to (3.2). For approximations of the Dirichlet problem (2.1) with reasonably fine meshes, the exact integration of the penalty term leads to so-called 'locked' solutions, and for a fixed mesh size $h$ such locked solutions will have the property that $\left\|\boldsymbol{u}_{\varepsilon}^{h}\right\|_{1}^{2}=O(\varepsilon)$ : hence, the divergent-free solution obtained as $\varepsilon \rightarrow 0$ is $\boldsymbol{u}^{h} \equiv \mathbf{0}$.

To illustrate the problem, consider the case of a mesh of 4-node isoparametric $\left(Q_{1^{-}}\right)$ elements on a polygonal domain and suppose $\operatorname{div} \boldsymbol{u}_{\varepsilon}^{h} \operatorname{div} \boldsymbol{v}^{h}$ is integrated exactly (e.g., $2 \times 2$ Gaussian quadrature is used, $G=4$ ). For the corner element illustrated in Fig. 1 we will have

$$
\boldsymbol{u}_{\varepsilon}^{h}=\left\{\frac{1}{4} \boldsymbol{u}_{\varepsilon}^{3}(1+\xi)(1+\eta)\right\} \boldsymbol{i}+\left\{\frac{1}{4} v_{\varepsilon}^{3}(1+\xi)(1+\eta)\right\} \boldsymbol{j}
$$

where $i$ and $j$ are mutually-orthogonal unit basis vectors and

$$
\operatorname{div} \boldsymbol{u}_{\varepsilon}^{h}=\frac{1}{4} u_{\varepsilon}^{3}(1+\eta)+\frac{1}{4} v_{\varepsilon}^{3}(1+\xi) .
$$




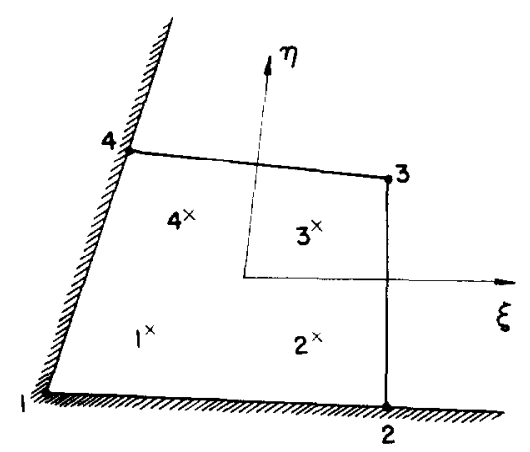

Fig. 1. Corner element.

Because of (4.3), both $u_{\varepsilon}^{3}$ and $v_{\varepsilon}^{3}$ are $\mathrm{O}(\sqrt{\varepsilon})$, and are almost zero for a sufficiently small $\varepsilon>0$. Thus, within the corner element, $\boldsymbol{u}_{\varepsilon}^{h}$ is 'locked'. Similar arguments can be made for adjacent elements, and we conclude that the entire solution is locked: $\boldsymbol{u}_{\varepsilon}^{h} \rightarrow 0$ as $\varepsilon \rightarrow 0$.

For the same element, however, if we under-integrate the penalty term by choosing only the one-point Gaussian quadrature rule for $I(\cdot, \cdot)$, then

$$
u_{\varepsilon}^{3}+v_{\varepsilon}^{3} \approx \mathrm{O}(\sqrt{\bar{\varepsilon}})
$$

i.e., $\boldsymbol{u}_{\varepsilon}^{h}$ need not be locked and nonzero solutions are possible.

Of course, one might avoid such difficulties by choosing non-uniform meshes with $h$ sufficiently small or, equivalently, limiting the reduction of $\varepsilon$ for a given mesh. But this is impractical: even for very fine meshes, $\varepsilon$ must typically be so large that the constraint $\operatorname{div} \boldsymbol{u}=0$ is not accurately satisfied.

Such locking phenomena and the necessity of selective reduced integration (i.e., the use of a quadraturc rulc $I$ of order less than that necessary for the exact integration of penalty terms) has been noted by many authors; see, for example, [20,35].

With these observations in mind, we now suppose that an 'unlocked' solution $\boldsymbol{u}_{\varepsilon}^{h}$ to (4.2) has been obtained and we proceed to define an approximation $p_{\varepsilon}^{h}$ of the hydrostatic pressure. We begin by introducing approximations $B_{h}$ and $B_{h}^{*}$ of the constraint operators $B$ and $B^{*}$ (which represent the divergence and gradient operators plus boundary conditions, respectively) defined by

$$
I\left(q^{h}, \operatorname{div} v^{h}\right)=\left(q^{h}, B_{h} v^{h}\right)=\left(B_{h}^{*} q^{h}, v^{h}\right)
$$

for every $v^{h} \in V_{h}$, and $q^{h} \in Q_{h}$. Here $Q_{h}$ is the finite-dimensional subspace of $Q$ which satisfies the following conditions:

(1) The numerical quadrature rule $I$ of (4.1) and the space $Q_{h}$ satisfy

$$
I\left(q^{h}, \operatorname{div} \boldsymbol{v}^{h}\right)=\left(q^{h}, \operatorname{div} \boldsymbol{v}^{h}\right), \quad \forall v^{h} \in V_{h}, \forall q^{h} \in Q_{h} .
$$

(2) There exists an element $p_{\varepsilon}^{h} \in Q_{h}$ such that

$$
p_{\varepsilon}^{h}\left(\boldsymbol{\xi}_{i}^{e}\right)=-\frac{1}{\varepsilon} \operatorname{div} \boldsymbol{u}_{\varepsilon}^{h}\left(\boldsymbol{\xi}_{i}^{e}\right)
$$


for $1 \leqslant i \leqslant G, 1 \leqslant e \leqslant E$, and $\left(p_{\varepsilon}^{h}\right)^{*} \in \operatorname{ker} B_{h}^{*}$, where

$$
\operatorname{ker} B_{h}^{*}=\left\{q^{h} \in Q_{h}: I\left(q^{h}, \operatorname{div} v^{h}\right)=0, \forall v^{h} \in V_{h}\right\}
$$

(3) There exists a positive number $\alpha_{h}$ such that

$$
\alpha_{h} \| q^{h} \eta_{0} \leqslant \sup _{v^{h} \in V_{h}-\{0\}} \frac{I\left(q^{h}, \operatorname{div} v^{h}\right)}{\left\|v^{h}\right\|_{1}}, \forall q^{h} \in Q_{h}
$$

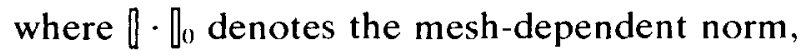

$$
\left\|q^{h}\right\|_{0}=\inf _{\left(q^{h}\right)^{*} \in \operatorname{ker} B_{h}^{*}}\left\|q^{h}+\left(q^{h}\right)^{*}\right\|_{0}
$$

For example, if $Q_{1}$-elements are employed and the one-point Gaussian quadrature rule $(G=1)$ is used to construct $I(\cdot, \cdot)$, conditions (1) and (2) imply that

$$
Q_{h}=\left\{q^{h}\left|q^{h}\right|_{\Omega_{e}}=\text { constant, } 1 \leqslant e \leqslant E, \text { and } \sum_{e} q_{e}^{h} \operatorname{meas}\left(\Omega_{e}\right)=0\right\} .
$$

Similarly, if 9-node isoparametric $\left(Q_{2^{-}}\right)$elements are used with $I(\cdot, \cdot)$ given by the $2 \times 2$ Gaussian quadrature rule, the space $Q_{h}$ for approximations of the pressure is characterized by (1) and (2) as the space spanned by globally-discontinuous functions which are bilinear polynomials over each element. On the other hand, if 6-node triangular $\left(P_{2^{-}}\right)$elements are employed for the velocity approximation with $I(\cdot, \cdot)$ given by a three-point quadrature rule, conditions (1) and (2) define as the space $Q_{h}$ a class of discontinuous piecewise linear functions,

$$
Q_{h}=\left\{q^{h}\left|q^{h}\right|_{\Omega_{e}} \in P_{1}\left(\Omega_{e}\right), 1 \leqslant e \leqslant E, \text { and } \int_{\Omega} q^{h} \mathrm{~d} x=0\right\}
$$

These examples are illustrated in Fig. 2. It is clear that the reduced-integration-penalty method characterized by (4.2) and conditions (1)-(3) above is equivalent to a regularized mixed finite-element method based on the perturbed-Lagrangian formulation (3.6) in which nonconforming (discontinuous) polynomial approximations of the pressures are employed. More specificially, when (1) and (2) hold, problem (4.2) is equivalent to the discrete problem,

$$
\begin{aligned}
& \left(\boldsymbol{u}_{\varepsilon}^{h}, p_{\varepsilon}^{h}\right) \in V_{h} \times Q_{h}, \\
& a\left(\boldsymbol{u}_{\varepsilon}^{h}, \boldsymbol{v}^{h}\right)-\left(p_{\varepsilon}^{h}, \operatorname{div} v^{h}\right)=f\left(\boldsymbol{v}^{h}\right), \quad \forall v^{h} \in V_{h}, \\
& \left(q^{h}, \varepsilon p_{\varepsilon}^{h}+\operatorname{div} \boldsymbol{u}_{\varepsilon}^{h}\right)=0, \quad \forall q^{h} \in Q_{h} .
\end{aligned}
$$

This equivalence has been observed and discussed by Malkus and Hughes [14, 21, 22].

We also note that by a straightforward (but lengthy) calculation of

$$
I\left(q^{h}, \operatorname{div} v^{h}\right)=0, \quad \forall v^{h} \in V_{h}
$$

for cach of thcse three examples, we can characterize the kernel of the discrete operator $B_{h}^{*}$. 

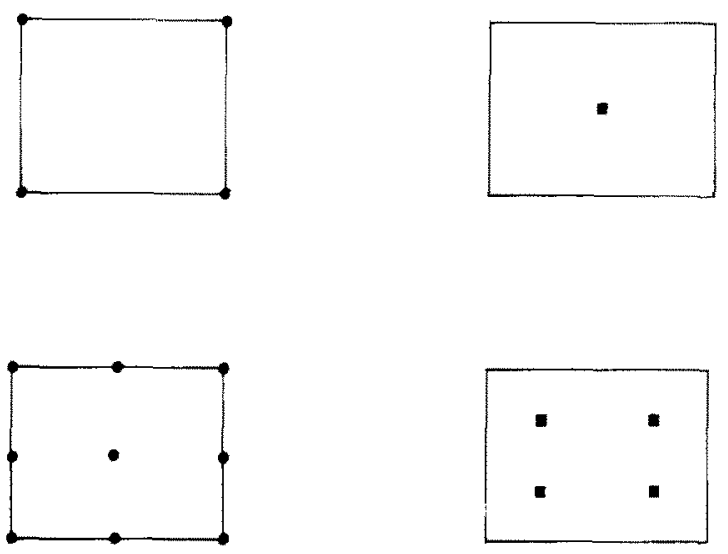

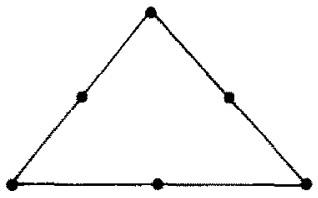

(a)

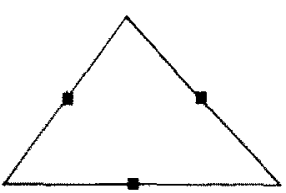

(b)
Fig. 2. Typical choices of $V_{h}$ and $Q_{h}$ : (a) velocity field; (b) hydrostatic pressure. (a)
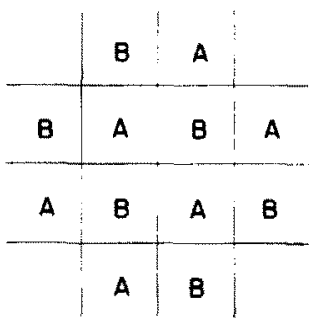

(b)

\begin{tabular}{ll|ll|ll}
$B$ & $A$ & $B$ & $A$ & $B$ & $A$ \\
\hline$A$ & $B$ & $A$ & $B$ & $A$ & $B$ \\
$B$ & $A$ & $B$ & $A$ & $B$ & $A$ \\
\hline$A$ & $B$ & $A$ & $B$ & $A$ & $B$
\end{tabular}

(c)

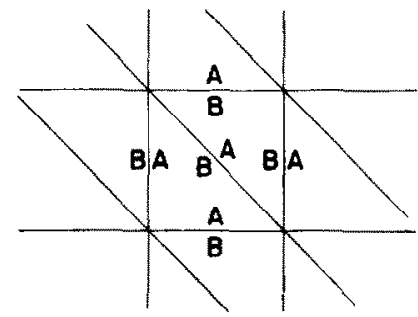

Fig. 3. Examples of $\operatorname{ker} B_{h}^{*}$ : (a) $Q_{1}$-elements 1-point Gaussian; (b) $Q_{2}$-elements $2 \times 2$-Gaussian; (c) $P_{2}$-elements 3-points Gaussian.

For example, in the case of a rectangular domain and a uniform mesh, $\operatorname{ker} B_{h}^{*}$ is $\left\{c, c_{b}(x)\right\}$, wherc $c$ is a constant and $c_{b}$ is the piccewise-constant checkerboard pattern shown in Fig. 3a. For $Q_{2}$-elements with $2 \times 2$-Gaussian quadrature, $\operatorname{ker} B_{h}^{*}$ contains an 'hour-glass' function which is piecewise bilinear over each element and which assumes the constant values $A$ or $B$ at the Gauss-points of each element (Fig. 3b). For the triangular $P_{2}$-elements with discontinuous piecewise-linear pressures, $\operatorname{ker} B_{h}^{*}$ contains constants and a discontinuous piecewise linear function which assumes the values $A$ or $B$ at the midpoints of sides of each triangle to form the pattern indicated in Fig. 3c.

REMARK 4.2 (One-point methods). The reduced-integration penalty methods described up to this point do not include cases in which the velocities are approximated by quadratic of higher-degree polynomials but $I(\cdot, \cdot)$ is defined by a simple one-point quadrature rule. Nevertheless, conclusions similar to those given above hold for such schemes with some minor modifications. For example, if $V_{h}$ is constructed using $Q_{2}$-elements, and $\left(q^{h}, \varepsilon p_{\varepsilon}^{h}+\operatorname{div} \boldsymbol{u}_{e}^{h}\right)$ is computed using piecewise constants $q^{h}$ and $p_{\varepsilon}^{h}$ on each element, $p_{\varepsilon}^{h}$ is defined by

$$
\left.p_{\varepsilon}^{h}\right|_{\Omega_{e}}=-\frac{\varepsilon^{-1}}{\operatorname{meas} \Omega_{e}} \sum_{j=1}^{G} W_{j}^{e} \operatorname{div} u_{\varepsilon}^{h}\left(\xi_{j}^{e}\right)
$$


with $G>0$. The corresponding penalty formulation is

$$
\boldsymbol{u}_{\varepsilon}^{h} \in V_{h}: \quad a\left(\boldsymbol{u}_{\varepsilon}^{h}, \boldsymbol{v}^{h}\right)+\varepsilon^{-1} \bar{I}\left(\operatorname{div} \boldsymbol{u}_{\varepsilon}^{h}, \operatorname{div} v^{h}\right)=f\left(v^{h}\right), \quad \forall v^{h} \in V_{h}
$$

where

$$
\bar{I}\left(\operatorname{div} \boldsymbol{u}_{\varepsilon}^{h}, \operatorname{div} \boldsymbol{v}^{h}\right)=\sum_{e=1}^{E} \frac{1}{\operatorname{meas} \Omega_{e}} \sum_{i=1}^{G} W_{i}^{e} \operatorname{div} \boldsymbol{u}_{\varepsilon}^{h}\left(\xi_{i}^{e}\right) \cdot \sum_{j=1}^{G} W_{j}^{e} \operatorname{div} v^{h}\left(\xi_{j}^{e}\right)
$$

If $2 \times 2$-(4-point) Gaussian quadrature is taken in (4.14), the constraint div $\boldsymbol{u}=0$ is satisfied in an average sense in each element as $\varepsilon \rightarrow 0$; i.e.,

$$
\frac{1}{4} \sum_{i=1}^{4} \operatorname{div} \boldsymbol{u}_{\varepsilon}^{h}\left(\xi_{i}^{e}\right) \rightarrow 0 \quad \text { as } \varepsilon \rightarrow 0
$$

for each element $\Omega_{e}$. Similar results hold for triangular $\left(P_{2^{-}}\right)$elements and one-point integration.

It is noteworthy that for the $Q_{2}$ and $P_{2}$-elements with the above formulation, we will have

$$
\operatorname{ker} B_{h}^{*} \subset \operatorname{ker} B^{*} \subset Q .
$$

Indeed, $\operatorname{ker} B_{h}^{*}=\operatorname{ker} B^{*}=\{0\}$. This relation does not hold, in general, for the other penaltyelements considered earlier.

We shall describe some additional properties of this class of penalty methods in Section 7.

\section{Convergence of $\left(u_{\varepsilon}^{h}, p_{\varepsilon}^{h}\right)$ to $(u, p)$}

If conditions (1)-(3) of the previous section hold, then it follows from arguments similar to those used in the proof of Theorem 3.1 that the sequence $\left\{\left(u_{\varepsilon}^{h}, p_{\varepsilon}^{h}\right)\right\} \in V_{h} \times Q_{h}$ of approximate solutions of (4.2), or equivalently (4.11), converges to a pair $\left(\boldsymbol{u}^{h}, p^{h}\right) \in V_{h} \times Q_{h}$ satisfying

$$
a\left(\boldsymbol{u}^{h}, \boldsymbol{v}^{h}\right)-\left(p^{h}, \operatorname{div} \boldsymbol{v}^{h}\right)=f\left(\boldsymbol{v}^{h}\right), \quad \forall \boldsymbol{v}^{h} \in V_{h}, \quad\left(q^{h}, \operatorname{div} \boldsymbol{u}^{h}\right)=0, \quad \forall q^{h} \in Q_{h}
$$

as $\varepsilon \rightarrow 0$. It is noted that the 'mixed' formulation (5.1) may not have a unique solution $\left(u^{h}, p^{h}\right)$ in $V_{h} \times Q_{h}$, since in some cases $\operatorname{ker} B_{h}^{*} \neq\{0\}$. However, the sequence $\left(\boldsymbol{u}_{\varepsilon}^{h}, p_{\varepsilon}^{h}\right)$ obtained by the penalty method converges to one of the solutions of (5.1) as $\varepsilon \rightarrow 0$. This proof is similar to the one for Theorem 3.1, but is different from it.

THEOREM 5.1. Let $\left(u_{\varepsilon}^{h}, p_{\varepsilon}^{h}\right)$ and $\left(u^{h}, p^{h}\right)$ be solutions of (4.11) and (5.1), respectively, and let the conditions (1)-(3) hold. Then

and

$$
\left\|u_{\varepsilon}^{h}-u^{h}\right\|_{1} \leqslant C \alpha_{h}^{-1} \varepsilon
$$

$$
\llbracket p_{\varepsilon}^{h}-p^{h} \rrbracket_{0} \leqslant 2 \mu C \alpha_{h}^{-2} \varepsilon .
$$


PROOF. From (4.11) and (5.1), we have

$$
a\left(\boldsymbol{u}_{\varepsilon}^{h}-\boldsymbol{u}^{h}, \boldsymbol{v}^{h}\right)=\left(p_{\varepsilon}^{h}-p^{h}, \operatorname{div} v^{h}\right), \quad \forall v \in V_{h}
$$

An application of (4.9) yields

$$
\left\|p_{\varepsilon}^{h}-p^{h} \coprod_{0} \leqslant\left(2 \mu / \alpha_{h}\right)\right\| \boldsymbol{u}_{\varepsilon}^{h}-\boldsymbol{u}^{h} \|_{1} .
$$

Setting $v^{h}=\boldsymbol{u}_{\varepsilon}^{h}-\boldsymbol{u}^{h}$ in (5.4), we obtain

$$
\begin{aligned}
2 \mu\left\|\boldsymbol{u}_{\varepsilon}^{h}-\boldsymbol{u}^{h}\right\|_{1}^{2} & \leqslant a\left(\boldsymbol{u}_{\varepsilon}^{h}-\boldsymbol{u}^{h}, \boldsymbol{u}_{\varepsilon}^{h}-\boldsymbol{u}^{h}\right) \leqslant\left(p_{\varepsilon}^{h}-p^{h}, \operatorname{div} \boldsymbol{u}_{\varepsilon}^{h}\right) \\
& =\left(p_{\varepsilon}^{h}-p^{h}+q^{h}, \operatorname{div} \boldsymbol{u}_{\varepsilon}^{h}\right), \quad \forall q^{h} \in \operatorname{ker} B_{h}^{*}
\end{aligned}
$$

since $\operatorname{div} \boldsymbol{u}^{h}=0$ in $L^{2}(\Omega)$. Noting that $q^{h}$ leads to the quotient norm $\rrbracket \cdot \rrbracket_{0}$ in the estimate (5.5), and setting $\operatorname{div} \boldsymbol{u}^{h}=-\varepsilon p_{\varepsilon}^{h}$, we have

$$
2 \mu\left\|\boldsymbol{u}_{\varepsilon}^{h}-\boldsymbol{u}^{h}\right\|_{1}^{2} \leqslant\left\|p_{\varepsilon}^{h}-p^{h} \eta_{0}\right\| p_{\varepsilon}^{h} \|_{0} \varepsilon
$$

Because of the manner of identification of the hydrostatic pressure $p_{\varepsilon}^{h}$ in (4.11),

$$
\left(q^{h}, p_{\varepsilon}^{h}+\frac{1}{\varepsilon} \operatorname{div} \boldsymbol{u}_{\varepsilon}^{h}\right)=\left(q^{h}, p_{\varepsilon}^{h}\right)=0, \quad \forall q^{h} \in \operatorname{ker} B_{h}^{*}
$$

Then the orthogonal relation (5.7) implies

$$
\left\|p_{\varepsilon}^{h}\right\|_{0}=\inf _{q^{h} \in \operatorname{ker} B_{h}^{*}}\left\|p_{\varepsilon}^{h}+q^{h}\right\|_{0}=\inf _{q^{h} \in \operatorname{ker} B_{h}^{*}}\left\{\left\|p_{\varepsilon}^{h}\right\|_{0}^{2}+\left\|q^{h}\right\|_{0}^{2}\right\}^{1 / 2}=\left\|p_{\varepsilon}^{h}\right\|_{0} .
$$

By using (4.9) we have

$$
\boldsymbol{\alpha}_{h}\left\|p_{\varepsilon}^{h}\right\|_{0} \leqslant \sup _{v^{h} \in V_{h}} \frac{a\left(\boldsymbol{u}_{\varepsilon}^{h}, \boldsymbol{v}^{h}\right)}{\left\|\boldsymbol{v}^{h}\right\|_{1}} \leqslant 2 \mu\left\|\boldsymbol{u}_{\varepsilon}^{h}\right\|_{1}
$$

Since $\left\|\boldsymbol{u}_{\varepsilon}^{h}\right\|_{1}$ is uniformly bounded in $\varepsilon$ and $h$,

$$
\left\|p_{\varepsilon}^{h}\right\|_{0} \leqslant C / \alpha_{h}
$$

for a proper positive number $C>0$.

Combining (5.5), (5.6) and (5.9), it is possible to conclude the results (5.2) and (5.9).

We emphasize that if $\operatorname{ker} B_{h}^{*} \not \subset \operatorname{ker} B^{*}$, then the norm $] \cdot \prod_{0}$ is different from $\|\cdot\|_{0}$, the former obviously depending on the mesh.

Another observation regarding the mixed problem (5.1) is that condition (3) (the discretc LBB-condition) and (5.1) imply that 


$$
\boldsymbol{\alpha}_{h} \llbracket p^{h} \prod_{0} \leqslant \sup _{\boldsymbol{v}^{h} \in V_{h}-\{0\}} \frac{\left(p^{h}, \operatorname{div} \boldsymbol{u}^{h}\right)}{\left\|\boldsymbol{v}^{h}\right\|_{1}} \leqslant 2 \mu\left\|\boldsymbol{u}^{h}\right\|_{1}+\|\boldsymbol{f}\|_{0} .
$$

In other words, a proper constant $M>0$ exists such that

$$
\| p^{h} \rrbracket_{0} \leqslant M / \alpha_{h}
$$

Thus, in view of (5.2) and (5.3), if the parameter $\alpha_{h}$ in the discrete LBB-condition (4.9) depends upon the mesh size $h$, it may be necessary to choose a very small $\varepsilon\left(=\mathrm{O}\left(\alpha_{h}^{3}\right)\right)$ in order to assure for convergence of the penalty solution $\left(u_{\varepsilon}^{h}, p_{\varepsilon}^{h}\right)$ to the solution of the corresponding mixed formulation as $h \rightarrow 0$.

Next, we shall consider the convergence of $\left(u^{h}, p^{h}\right) \in V_{h} \times Q_{h}$ to the solution $(u, p) \in V \times Q$ of the Stokes' problem (2.1).

THEOREM 5.2. Suppose that the conditions (1)-(3) hold. Then constants $M_{1}$ and $M_{2}$ exist, independent of $h$, such that

$$
\left\|\boldsymbol{u}^{h}-\boldsymbol{u}\right\|_{1} \leqslant M_{1}\left(1+\frac{1}{\alpha_{h}}\right)\left(\left\|v^{h}-\boldsymbol{u}\right\|_{1}+\left|p-q^{h}\right|_{0}\right)
$$

and

$$
\| p^{h}-p \llbracket_{0} \leqslant M_{2}\left(1+\frac{1}{\alpha_{h}}+\frac{1}{\alpha_{h}^{2}}\right)\left(\left|q^{h}-p\right|_{0}+\left\|v^{h}-u\right\|_{1}\right)
$$

for all $v^{h} \in V_{h}$ and $q^{h} \in Q_{h}$, where $\left(u^{h}, p^{h}\right)$ is the solution of (5.1) and $(u, p)$ is the solution of (2.1).

PROOF. Setting $v=v^{h}$ in (2.1) and subtracting from (5.1) yields

$$
a\left(\boldsymbol{u}^{h}-\boldsymbol{u}, \boldsymbol{v}^{h}\right)=\left(p^{h}-p, \operatorname{div} v^{h}\right), \quad \forall v^{h} \in V_{h} .
$$

Then

$$
a\left(\boldsymbol{u}^{h}-\boldsymbol{u}, \boldsymbol{u}^{h}-\boldsymbol{u}\right)=\left(p^{h}-p, \operatorname{div}\left(\boldsymbol{u}^{h}-\boldsymbol{v}\right)\right)+a\left(\boldsymbol{u}^{h}-\boldsymbol{u}, \boldsymbol{v}^{h}-\boldsymbol{u}\right)
$$

Since $\left(q^{h}, \operatorname{div} \boldsymbol{u}^{h}\right)=\left(q^{h}, \operatorname{div} \boldsymbol{u}\right)=0, \forall q^{h} \in Q_{h}$, we have

$$
\left(p^{h}-p, \operatorname{div}\left(\boldsymbol{u}^{h}-v^{h}\right)\right)=\left(p-q^{h}, \operatorname{div}\left(v^{h}-\boldsymbol{u}^{h}\right)\right)+\left(p^{h}-q^{h}, \operatorname{div}\left(u-v^{h}\right)\right) .
$$

Thus, for arbitrary $v^{h} \in V_{h}$ and $q^{h} \in Q_{h}$,

$$
\begin{aligned}
a\left(\boldsymbol{u}^{h}-\boldsymbol{u}, \boldsymbol{u}^{h}-\boldsymbol{u}\right)= & a\left(\boldsymbol{u}^{h}-\boldsymbol{u}, \boldsymbol{v}^{h}-\boldsymbol{u}\right)+\left(p-q^{h}, \operatorname{div}\left(\boldsymbol{u}-\boldsymbol{u}^{h}\right)\right)+\left(p-q^{h}, \operatorname{div}\left(\boldsymbol{v}^{h}-\boldsymbol{u}\right)\right) \\
& +\left(p^{h}-q^{h}, \operatorname{div}\left(\boldsymbol{u}-\boldsymbol{v}^{h}\right)\right) .
\end{aligned}
$$

On the other hand, the discrete LBB-condition (4.9) implies that 
i.e.,

$$
\begin{aligned}
\alpha_{h} \rrbracket q^{h}-p^{h} \prod_{0} & \leqslant \sup _{v^{h} \in V_{h}-\{0\}} \frac{\left(q^{h}-p^{h}, \operatorname{div} \boldsymbol{v}^{h}\right)}{\left\|\boldsymbol{v}^{h}\right\|_{1}} \\
& =\sup _{\boldsymbol{v}^{h} \in V_{h}-\{0\}} \frac{\left(q^{h}-p, \operatorname{div} \boldsymbol{v}^{h}\right)+a\left(\boldsymbol{u}-\boldsymbol{u}^{h}, \boldsymbol{v}^{h}\right)}{\left\|\boldsymbol{v}^{h}\right\|_{1}} \\
& \leqslant\left\|q^{h}-p\right\|_{0}+2 \mu\left\|\boldsymbol{u}-\boldsymbol{u}^{h}\right\|_{1},
\end{aligned}
$$

$$
\emptyset q^{h}-p^{h} \rrbracket_{0} \leqslant \frac{1}{\alpha_{h}}\left(\left\|q^{h}-p\right\|_{0}+2 \mu\left\|\boldsymbol{u}-\boldsymbol{u}^{h}\right\|_{1}\right)
$$

From (5.13) and (5.14) it is easily established that a proper positive constant $c>0$ exists such that

$$
c\left\|\boldsymbol{u}^{h}-\boldsymbol{u}\right\|_{1}^{2} \leqslant\left\|\boldsymbol{v}^{h}-\boldsymbol{u}\right\|_{1}^{2}+\left\|p-q^{h}\right\|_{0}^{2}+\left(\frac{1}{\alpha_{h}}\right)^{2}\left(\left\|q^{h}-p\right\|_{0}^{2}+\left\|\boldsymbol{v}^{h}-\boldsymbol{u}\right\|_{1}^{2}\right),
$$

i.e., the estimate (5.10) follows from this result. On the other hand,

$$
\rrbracket p^{h}-p \rrbracket_{0} \leqslant \rrbracket p^{h}-q^{h} \rrbracket_{0}+\rrbracket q^{h}-p \rrbracket_{0} \leqslant \rrbracket p^{h}-q^{h} \rrbracket_{0}+\left\|q^{h}-p\right\|_{0} .
$$

Thus (5.14) and this result imply (5.11).

\section{The discrete LBB-condition}

In this section, we attempt to evaluate the stability parameters $\alpha_{h}$ appearing in the discrete LBB-condition (4.9) for the approximation of the Stokes' problem. We first consider the stability condition (4.9) for two of the most popular elements: $Q_{1}$-(bilinear) elements with 1-point integration and $Q_{2}$-(biquadratic) elements with $2 \times 2$-(4-point) Gaussian quadrature. Our numerical experiments indicate that these elements are marginally stable: especially the hydrostatic pressure $p_{\varepsilon}^{h}$ is very sensitive to mild singularities in the applied forces and boundary conditions. But on rectangular uniform meshes they may yield surprising good results for velocities when the solution is smooth enough. For these elements, ker $B_{h}^{*} \not \subset$ ker $B^{*}$, and ker $B_{h}^{*}$ contains the notorious checkerboard patterns described earlier (recall Fig. 3).

Throughout this part of our analysis we confine ourselves to a uniform mesh of rectangular elements defined on a rectangular domain in $\mathbb{R}^{2}$. The space $V_{h}$ is obtained by partitioning $\Omega$ into a uniform mesh of $E$ rectangular elements $\bar{\Omega}_{e}$ of diameter $h$, and we consider only regular uniform refinements of the mesh. In particular, we will choose $V_{h}$ to be $V_{h}^{(1)}$ or $V_{h}^{(2)}$, where

$$
\begin{aligned}
V_{h}^{(s)}=\left\{\boldsymbol{v}^{h}=\left(v_{1}^{h}, v_{2}^{h}\right) \mid v_{i}^{h}\right. & \in C^{0}(\bar{\Omega}),\left.v_{i}^{h}\right|_{\bar{\Omega}_{e}} \in Q_{s}\left(\bar{\Omega}_{e}\right), \\
\boldsymbol{v}^{h} & \left.=\mathbf{0} \text { on } \Gamma_{D}, 1 \leqslant e \leqslant E, i=1,2\right\}, \quad s=1,2 .
\end{aligned}
$$

Here $Q_{1}$ and $Q_{2}$ are the spaces of tensor products of polynomials of degree 1 and 2 , respectively. 
We first introduce the following algebraic results for later use.

LEMMA 6.1. Let $\left\{a_{i}\right\}_{i=1}^{r}$ be a set of real numbers. Then the following inequality holds,

$$
a_{1}^{2}+\sum_{i=2}^{r}\left(a_{i-1}-a_{i}\right)^{2} \geqslant \frac{1}{m} a_{m}^{2}, \quad \forall m, m=1, \ldots, r .
$$

PROOF. We first note for any given $\alpha>0$,

(a) $\quad \alpha a_{i}^{2}+\left(a_{i}-a_{j}\right)^{2} \geqslant \frac{\alpha}{1+\alpha} a_{j}^{2}$.

We then prove (6.2) by induction. We assume that the following holds:

(b) $\quad a_{1}^{2}+\sum_{i=2}^{r}\left(a_{i-1}-a_{i}\right)^{2} \geqslant \frac{1}{m} a_{m}^{2}+\sum_{i=m+1}^{r}\left(a_{i-1}-a_{i}\right)^{2}$.

Clearly (b) holds for $m=1$. If it also is true for $m=n$, then

$$
\begin{aligned}
a_{1}^{2}+\sum_{i=2}^{r}\left(a_{i-1}-a_{i}\right)^{2} & \geqslant \frac{1}{n} a_{n}^{2}+\sum_{i=n+1}^{r}\left(a_{i-1}-a_{i}\right)^{2} \\
& =\frac{1}{n} a_{n}^{2}+\left(a_{n}-a_{n+1}\right)^{2}+\sum_{i=n+2}^{r}\left(a_{i-1}-a_{i}\right)^{2} \\
& \geqslant \frac{1 / n}{1+1 / n} a_{n+1}^{2}+\sum_{i=n+2}^{r}\left(a_{i-1}-a_{i}\right)^{2} \\
& =\frac{1}{n+1} a_{n+1}^{2}+\sum_{i=n+2}^{r}\left(a_{i-1}-a_{i}\right)^{2} .
\end{aligned}
$$

Thus (b) is true for $m=n+1$. Hence (b) is valid. Then (6.2) is direct consequence.

LEMMA 6.2. Let $\left\{a_{i}\right\}_{i=1}^{N}$ be a set of real numbers. Then the following inequality holds:

$$
a_{1}^{2}+\sum_{i=2}^{N}\left(a_{i-1}-a_{i}\right)^{2} \geqslant \frac{1}{N^{2}} \sum_{i=1}^{N} a_{i}^{2} .
$$

PROOF. By (6.2),

Thus,

$$
a_{1}^{2}+\sum_{i=2}^{N}\left(a_{i-1}-a_{i}\right)^{2} \geqslant \frac{1}{r} a_{r}^{2}, \quad r=1, \ldots, N .
$$

$$
N\left(a_{1}^{2}+\sum_{i=2}^{N}\left(a_{i-1}-a_{i}\right)^{2}\right) \geqslant \sum_{r=1}^{N} \frac{1}{r} a_{r}^{2} \geqslant \frac{1}{N} \sum_{i=1}^{N} a_{i}^{2} .
$$


Dividing both sides of the above inequality by $N$, we obtain (6.3).

THEOREM 6.3 (4-node, $Q_{1}$-elements). Let the domain $\bar{\Omega}$ be rectangular and every $v^{h} \in V_{h}$ be such that $v^{h}=\mathbf{0}$ on $\Gamma$. Thus, for $Q_{h}=\left\{q^{h}\left|q^{h}\right|_{\bar{\Omega}_{e}}=\right.$ constant, $1 \leqslant e \leqslant E,\left.\Sigma_{e} p^{h}\right|_{\Omega_{e}}$ meas $\left.\left(\Omega_{e}\right)=0\right\}$ and $V_{h}=V_{h}^{(1)}$, the LBB-constant $\alpha_{h}$ satisfying

$$
\alpha_{h} \rrbracket q^{h} \prod_{0} \leqslant \sup _{\boldsymbol{v}^{h} \in v_{h}} \frac{I\left(q^{h}, \operatorname{div} \boldsymbol{v}^{h}\right)}{\left\|\boldsymbol{v}^{h}\right\|_{1}}\left(\boldsymbol{v}^{h} \neq \mathbf{0}\right) \forall q^{h} \in Q_{h}
$$

is of order $h^{2}: \alpha_{h}=\mathrm{O}\left(h^{2}\right)$. [An improvement $\mathrm{O}(h)$ is possible; see the addendum at the end of this paper.]

PROOF. In this case, $G=1$ in the definition of $I(\cdot, \cdot)$ and

$$
\begin{aligned}
I\left(q^{h}, \operatorname{div} \boldsymbol{v}^{h}\right) & =\sum_{e=1}^{E} I_{e}\left(q^{h}, \operatorname{div} \boldsymbol{v}^{h}\right)=h^{2} \sum_{e=1}^{E} q^{h}\left(\boldsymbol{\xi}^{e}\right) \operatorname{div} \boldsymbol{v}^{h}\left(\boldsymbol{\xi}^{e}\right) \\
& =\frac{1}{2} h \sum_{i=1}^{N}\left\{v_{1 i}\left(q_{i}^{1}-q_{i}^{2}-q_{i}^{3}+q_{i}^{4}\right)+v_{2 i}\left(q_{i}^{1}+q_{i}^{2}-q_{i}^{3}-q_{i}^{4}\right)\right\}
\end{aligned}
$$

where $E$ is the number of elements, $N$ the number of nodes, $\xi^{e}$ are the Gauss points in $\Omega_{e}, q_{i}^{n}$ the constant pressures in quadrant $n$ of a collection of four elements meeting at node $i$ numbered as shown in Fig. 4.

For fixed $q^{h}$ we choose $v^{h} \in V_{h}$ such that

Then,

$$
v_{1 i}=q_{i}^{1}-q_{i}^{2}-q_{i}^{3}+q_{i}^{4}, \quad v_{2 i}=q_{i}^{1}+q_{i}^{2}-q_{i}^{3}-q_{i}^{4} .
$$

$$
I\left(q^{h}, \operatorname{div} v^{h}\right)=h\left\{\sum_{i=1}^{N}\left(v_{1 i}^{2}+v_{2 i}^{2}\right)\right\}^{1 / 2}\left\{\sum_{i=1}^{N} 2\left[\left(q_{i}^{1}-q_{i}^{3}\right)^{2}+\left(q_{i}^{2}-q_{i}^{4}\right)^{2}\right]\right\}^{1 / 2}
$$

If $\bar{q}^{h} \in$ ker $B_{h}^{*}$, a series of calculations similar to those above gives

$$
I\left(q^{h}, \operatorname{div} v^{h}\right)=h\left\{\sum_{i=1}^{N}\left(v_{1 i}^{2}+v_{2 i}^{2}\right)\right\}^{1 / 2}\left\{\sum_{i=1}^{N} 2\left[\left(\hat{q}_{i}^{1}-\hat{q}_{i}^{3}\right)^{2}+\left(\hat{q}_{i}^{2}-\hat{q}_{i}^{4}\right)^{2}\right]\right\}^{1 / 2}
$$

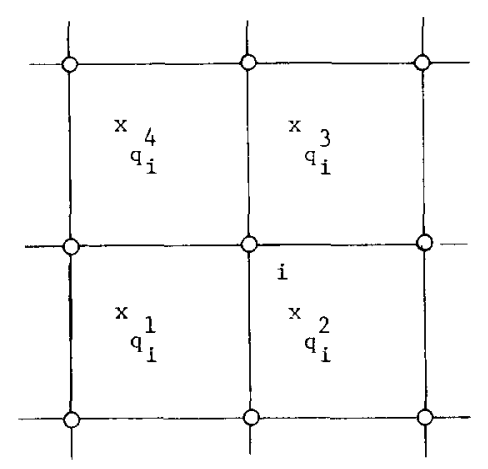

Fig. 4. $Q_{1}$ elements with 1-point integration. 
for $q^{h}=\hat{q}^{h}+\bar{q}^{h}$. Taking the special $\bar{q}^{h} \in \operatorname{ker} B_{h}^{*}$ such that

yields

$$
\hat{q}_{1}^{1}=0 \quad \text { and } \quad \hat{q}_{1}^{2}=0
$$

$$
\sum_{i=1}^{N}\left[\left(\hat{q}_{i}^{1}-\hat{q}_{i}^{3}\right)^{2}+\left(\hat{q}_{i}^{2}-\hat{q}_{i}^{4}\right)^{2}\right] \geqslant C h^{4} \sum_{e=1}^{E} \hat{q}_{e}^{2} .
$$

Here we have used the fact that $\operatorname{ker} B_{h}^{*}$ consists of piecewise constant functions of the type $\bar{q}_{i}^{1}=\bar{q}_{i}^{3}=A$ and $\bar{q}_{i}^{2}=\bar{q}_{i}^{4}=B$ (because of the condition $\int_{\Omega} q \mathrm{~d} x=0$ in $Q, B$ must be $-A$ ), and that $N=\mathrm{O}\left(1 / h^{2}\right)$. Then (6.8) becomes

$$
I\left(q^{h}, \operatorname{div} \boldsymbol{v}^{h}\right) \geqslant h\left\{\sum_{i=1}^{N}\left(v_{1 i}^{2}+v_{2 i}^{2}\right)\right\}^{1 / 2}\left\{C h^{4} \sum_{e=1}^{E} \hat{q}_{e}^{2}\right\}^{1 / 2}
$$

By direct calculation we can show that

$$
\left\|\boldsymbol{v}^{h}\right\|_{1}^{2} \leqslant C \sum_{i=1}^{N}\left(v_{1 i}^{2}+v_{2 i}^{2}\right) \text { and } \| q^{h} \prod_{0}^{2} \leqslant C h^{2} \sum_{e=1}^{E} \hat{q}_{e}^{2} .
$$

Thus, $\alpha_{h}=C h^{2}=\mathrm{O}\left(h^{2}\right)$, as asserted.

Exactly the same procedure described in the proof of Theorem 6.3 can be used to study case of 9-node isoparametric element with $2 \times 2$ Gaussian rule for $I(\cdot)$. Hence we have the following theorem.

THEOREM 6.4 (9-node $Q_{2}$-elements). Let the domain $\Omega$ be rectangular and let $V_{h} \subset V$ be constructed using a uniform mesh of rectangular elements with $V_{h}=V_{h}^{(2)}$. Moreover, let $Q_{h}$ be defined by

$$
Q_{h}=\left\{q_{h}\left|q^{h}\right|_{\bar{\Omega}_{e}} \in Q_{1}\left(\bar{\Omega}_{e}\right), 1 \leqslant e \leqslant E, \int_{\Omega} q^{h} \mathrm{~d} x=0\right\} .
$$

Then the LBB-constant $\alpha_{h}$ of (4.9) for these spaces is such that $\alpha_{h}=\mathrm{O}\left(h^{2}\right)$.

It is now clear that convergence cannot be concluded from the results in Theorems 5.2, 6.3 and 6.4 for $Q_{1}$-elements with 1-point integration and $Q_{2}$-elements with $2 \times 2$-integration. Estimates in (5.5) and (5.6) suggest that in order to obtain convergence of $\left\|\boldsymbol{u}-\boldsymbol{u}^{h}\right\|_{1}$ and $\| p-p^{h} \rrbracket_{0}$ to zero as $h \rightarrow 0$, the LBB-constant $\alpha_{h}$ must be $O\left(h^{\beta}\right), \beta<\frac{1}{2}$. We note, however, that the above result does not imply the method of $Q_{1}$-elements with 1-point integration rule always diverges as $h \rightarrow 0$, since the estimate of $\alpha_{h}$ obtained above need not be optimal.

\section{Projection methods for the $Q_{2}$-element with '1-point' integration rule}

In the study of conditions similar to (4.9) for certain mixed methods, Crouzeix and Raviart [9], Girault and Raviart [12,13] and Fortin [10] have shown that, to prove the existence of $\alpha_{h}$ 
independent of $h$, it is sufficient to find a $w^{h}$ such that

$$
\left(\operatorname{div} \boldsymbol{w}^{h}-q^{h}, \hat{q}^{h}\right)=0, \quad \forall \hat{q}^{h} \in Q_{h}, \quad\left\|\boldsymbol{w}^{h}\right\|_{1} \leqslant C\left\|q^{h}\right\|_{0} .
$$

Indeed, it follows from (7.1) that for all $q^{h}$ in $Q_{h}$,

$$
\sup _{\boldsymbol{v}^{h} \in V_{h}} \frac{\left(q^{h}, \operatorname{div} v^{h}\right)}{\left\|\boldsymbol{v}^{h}\right\|_{1}} \geqslant \frac{1}{C}\left\|q^{h}\right\|_{0}
$$

Hence, (4.9) is satisfied with $\alpha_{h}=1 / C$ independent of $h$.

We now consider the penalty method (4.14) obtained by $Q_{2}$-elements and '1-point' integration rule discussed in Remark 4.2.

Let the mesh of rectangular elements be generated by a sequence of affine invertible maps of a master element $\hat{\Omega}$ in the spirit of Ciarlet and Raviart [7,8]. Indeed, let $V(\hat{\Omega})$ denote the 18-dimensional space of vector-valued function $\hat{\boldsymbol{V}}$ whose components are biquadratic polynomials, and let $F_{e}: \hat{\Omega} \rightarrow \Omega_{e}$ be invertible affine map of $\hat{\Omega}$ onto a typical element $\Omega_{e}$ in the mesh. For $\varphi=\varphi(x), x \in \Omega_{e}$, denote $\hat{\varphi}=\varphi \circ F_{e}$.

THEOREM 7.1. Let the families of spaces $\left\{V_{h}\right\}$ be constructed using uniform regular partitions of $\Omega$ into $C^{0}$-9-node quadratic rectangles $\left(Q_{2}\right.$-elements $)$. Let $\bar{I}(\cdot)$ be defined by ' 1 -point' Gaussian quadrature on rectangles as in (4.14). Then there exists $\alpha_{h}>0$, independent of $h$ such that (4.9) holds for $h$ sufficiently small.

PROOF. (1) Let $v \in V$ be the solution to the problem

$$
\operatorname{div} \boldsymbol{v}=q^{h} \text { in } \Omega, \quad\|v\|_{1} \leqslant C\left\|q^{h}\right\|_{0}
$$

for a given $q^{h} \in Q_{h} \subset Q$. The existence of such a $v$ is assured by the LBB-condition for the continuous problem. Suppose that $\boldsymbol{W}$ is the $V$-orthogonal projection of $v$ onto $V_{h}$ :

$$
\int_{\Omega}\left(v_{i}-W_{i}\right)_{, j} V_{i, j} \mathrm{~d} x=0, \quad \forall V \in V(\Omega) .
$$

Clearly, $\|\boldsymbol{W}\|_{1, \Omega} \leqslant\|\boldsymbol{v}\|_{1, \Omega}$.

(2) Let $\left\{a_{i}\right\}_{i=1}^{5}$ and $\left\{a_{i j}\right\}_{1 \leqslant i \leqslant j \leqslant 4}$ denote nodes on the master element numbered as in Fig. $5 \mathrm{~b}$. We pick an element $\hat{U} \in V(\hat{\Omega})$ such that

$$
\begin{aligned}
& \hat{U}_{i}\left(a_{j}\right)=W_{i}\left(a_{j}\right), \quad i=1,2 ; j=1,2,3,4,5, \\
& \hat{\boldsymbol{U}}\left(a_{i j}\right) \cdot \hat{\tau}\left(a_{i j}\right)=W\left(a_{i j}\right) \cdot \hat{\tau}\left(a_{i j}\right), \quad 1 \leqslant i \leqslant j \leqslant 4
\end{aligned}
$$

where $\hat{\boldsymbol{\tau}}$ is the unit vector tangent to $\partial \hat{\Omega}$.

(3) Conditions (7.4) provide 14 independent conditions for the 18 degrees of freedom of $\hat{\boldsymbol{U}}$. The remaining four conditions specify the normal components at the midside nodes: 


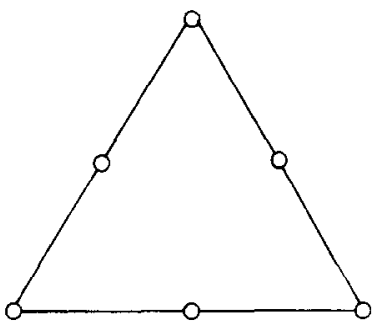

(a)

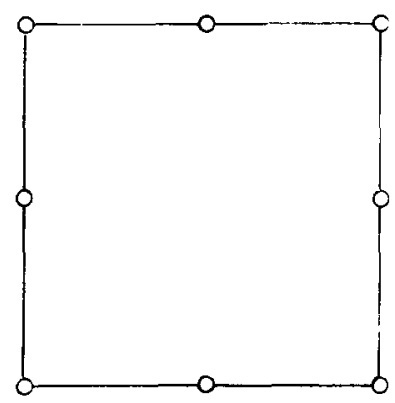

(c)

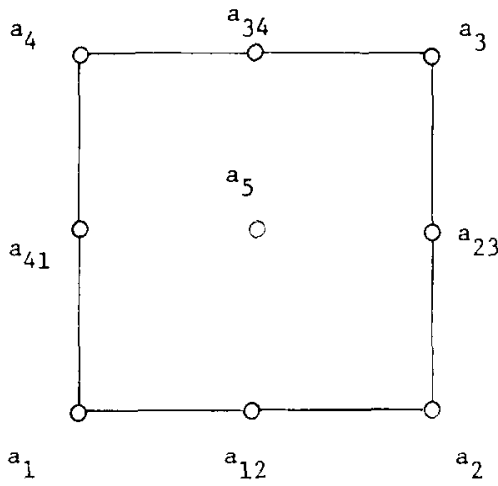

(b)

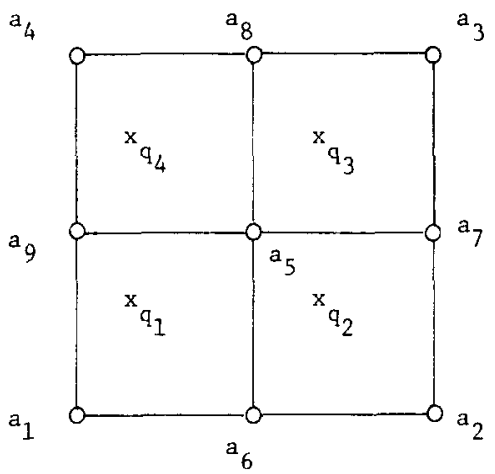

(d)

Fig. 5. Some two-dimensional biquadratic elements and a composite element consisted of four bilinear elements.

$$
\int_{a_{i}}^{a_{j}}\left(\hat{\boldsymbol{U}}\left(a_{i}\right) \cdot \hat{\boldsymbol{n}} \hat{\varphi}^{i}+\hat{\boldsymbol{U}}\left(a_{j}\right) \cdot \hat{\boldsymbol{n}} \hat{\varphi}^{j}-\hat{\boldsymbol{v}} \cdot \hat{\boldsymbol{n}}\right) \mathrm{d} \hat{\boldsymbol{s}}+\int_{a_{i}}^{a_{j}} \hat{\boldsymbol{U}}\left(a_{i j}\right) \cdot \hat{\boldsymbol{n}} \hat{\varphi}^{i j} \mathrm{~d} \hat{\boldsymbol{s}}=0
$$

Here $\hat{\varphi}^{i}, \hat{\varphi}^{i j}$ are the bilinear shape functions for nodes $a_{i}$ and $a_{i j}$ and $\hat{n}$ is the unit outward normal to $\partial \hat{\Omega}$.

(4) The unique function $\hat{U}$ constructed via (7.4) and (7.5) satisfies

$$
\oint_{\hat{\Omega}}(\hat{U}-\hat{v}) \cdot \hat{n} \hat{q} \mathrm{~d} \hat{s}=0=\int_{\hat{\Omega}} \operatorname{div}(\hat{U}-\hat{v}) \hat{q} \mathrm{~d} \hat{x}
$$

for every constant $q$.

(5) It remains to show that the second equation in (7.1) holds. For a uniform regular family of partitions of $\Omega$, we set $e_{h}=U-W, e=W-v$. Then, following the ideas of Girault and Raviart [12, Lemma 2.5, p. 76], we can show that $\left\|e_{h}\right\|_{1} \leqslant C h^{-1}\left(\int_{\Omega}|e|^{2} \mathrm{~d} x+\|e\|_{1}^{2}\right)^{1 / 2}$, from which we conclude that

$$
\|\hat{\boldsymbol{U}}\|_{1} \leqslant C(1+1 / h)\left\|q^{h}\right\|_{0}
$$


REMARK 7.2. The above proof employs the strategy used by Crouzeix and Raviart [9] for a mixed method obtained using 6-node $P_{2}$-elements with a piecewise constant pressure field.

Thus from the estimates in Section 5 and the theory of interpolation we have the following.

THEOREM 7.3. Let the conditions of Theorem 7.1 hold and suppose $\boldsymbol{u} \in\left(H^{2}(\Omega)\right)^{n} \cap V$ and $p \in H^{1}(\Omega) \cap Q^{2}$. Then the following estimate holds for some positive constant $C$ independent of $h$ :

$$
\left\|\boldsymbol{u}-\boldsymbol{u}^{h}\right\|_{1}+\left\|p-p^{h}\right\|_{0} \leqslant C\left(\|\boldsymbol{u}\|_{2}+\|p\|_{1}\right) h .
$$

We note that while this under-integrated scheme is stable $\left(\alpha_{h}\right.$ is independent of $h$ ), inexact integration has reduced the rate of convergence by one order lower than optimal. Our numerical experiments have confirmed this observation.

\section{A projection method for composite $Q_{1}$-elements}

The fact, proved in Section 6, that the $Q_{1}$-elements with 1-point Gaussian quadrature may lead to a stability parameter $\alpha_{h}=\mathrm{O}\left(h^{2}\right)$ indicates that schemes employing this element may be divergent. Nevertheless, this element is very popular in engineering computations because of its simplicity. We shall now describe a slight modification of this element which leads to a stable method which exhibits optimal rates of convergence. The construction of a special composite element is described as follows.

(P.1) Let $\Omega \subset \mathbb{R}^{2}$ be a rectangular domain. We partition $\Omega$ into a uniform mesh of rectangular elements $\left\{\Omega_{e}\right\}_{e=1}^{E}$ each consisting of four equal rectangular subelements $K_{e}^{i}$ :

$$
\bar{\Omega}=\bigcup_{e=1}^{E} \bar{\Omega}_{e} ; \quad \bar{\Omega}_{e}=\bigcup_{i=1}^{4} K_{e}^{i} .
$$

Over each subrectangle $K_{e}^{i}$ we approximate the components of the displacement vector $v^{h}$ by $C^{0}$-bilinear functions of $\boldsymbol{x}=\left(x_{1}, x_{2}\right)$. Thus, a composite element consisting of four bilinear $\left(Q_{1}\right)$ subelements of the type shown in Fig. 4 is obtained. With families of such elements, we obtain families $\left\{V_{h}\right\}$ of finite-dimensional subspaces of $V=\left(H_{0}^{1}(\Omega)\right)^{2}$ :

$$
\begin{gathered}
V_{h}=\left\{\boldsymbol{v}^{h}=\left(v_{1}^{h}, v_{2}^{h}\right) \in C^{0}(\bar{\Omega})\left|v_{j}^{h}\right|_{K_{e}^{i}} \in Q_{1}\left(K_{e}^{i}\right), j=1,2 ;\right. \\
i=1,2,3,4 ; \boldsymbol{e}=1,2, \ldots, E\} .
\end{gathered}
$$

(P.2) The approximation of pressures $q^{h}$ is constructed in two steps. First, we generate a family of finite-dimensional spaces $\tilde{Q}_{h}$ of piecewise constant pressures defined by

$$
\tilde{Q}_{h}=\left\{q^{h} \in Q^{2}(\Omega)\left|q^{h}\right|_{K_{e}^{i}} \equiv q_{e}^{i}=\text { constant, } i, 2,3,4 ; 1 \leqslant e \leqslant E\right\}
$$

where the numbering scheme shown in Fig. $5 \mathrm{~d}$ is used. This is preciscly the space used in Section 6 which led to the (unacceptable) stability parameter $\alpha_{h}=\mathrm{O}\left(h^{2}\right)$. 
We shall take for our space of approximate pressure,

$$
Q_{h}=\left\{q^{h} \in \tilde{Q}_{h} \mid q_{e}^{1}+q_{e}^{3}=q_{e}^{2}+q_{e}^{4}, 1 \leqslant e \leqslant E\right\} .
$$

A word of interpretation is called for. Consider a linear function $\bar{q}_{e}^{h}$ defined on $\bar{\Omega}_{e}$ of the form

$$
\bar{q}_{e}^{h}\left(x_{1}, x_{2}\right)-\bar{q}_{0}+\bar{q}_{1} x_{1}+\bar{q}_{2} x_{2}, \quad\left(x_{1}, x_{2}\right) \in \bar{\Omega}_{e} .
$$

Then, if the numbers $q_{e}^{i}$ are such that $\bar{q}_{e}^{h}\left(\xi_{e}^{i}\right)=q_{e}^{i}$ for the centroid $\boldsymbol{\xi}_{e}^{i}$ of each composite element, the function $\bar{q}_{e}^{h}$ will define a plane which passes through the four points $\left(\xi_{e}^{i}, q_{e}^{i}\right)$, $i=1,2,3,4$. Then we will have $q_{e}^{1}+q_{e}^{3}=q_{e}^{2}+q_{e}^{4}$.

Note also that for any $q^{h} \in Q_{h}$, we will have

$$
\begin{aligned}
& q_{e}^{1}-q_{e}^{2}=q_{e}^{4}-q_{e}^{3}, \quad q_{e}^{1}-q_{e}^{4}=q_{e}^{2}-q_{e}^{3}, \\
& \frac{q_{e}^{1}-q_{e}^{2}}{q_{e}^{1}-q_{e}^{2}-q_{e}^{3}+q_{e}^{4}}=\frac{q_{e}^{4}-q_{e}^{3}}{q_{e}^{1}-q_{e}^{2}-q_{e}^{3}+q_{e}^{4}}=\frac{q_{e}^{1}-q_{e}^{4}}{q_{e}^{1}+q_{e}^{2}-q_{e}^{3}-q_{e}^{4}}=\frac{q_{e}^{2}-q_{e}^{3}}{q_{e}^{1}+q_{e}^{2}-q_{e}^{3}-q_{e}^{4}}=\frac{1}{2} .
\end{aligned}
$$

Another significant feature of $Q_{h}$ is that if $B_{h}^{*}\left(\tilde{Q}_{h}\right)$ is the adjoint of the discrete operator associated with the space $\tilde{Q}_{h}$, then, in view of (6.6),

$$
Q_{h} \cap \operatorname{ker} B_{h}^{*}\left(\tilde{Q}_{h}\right)=\{0\} .
$$

Thus, the choice of $Q_{h}$ over $\tilde{Q}_{h}$ reduces $\operatorname{ker} B_{h}^{*}$ to the trivial set $\{0\}$. This means that if the condition $\int_{\Omega} g^{h} \mathrm{~d} x=0$ is applied in $Q_{h}$ as in $Q$, then $\operatorname{ker} B_{h}^{*}=\operatorname{ker} B^{*}$.

Within a composite element $\Omega_{e}$, we shall define the quantity

where

$$
\left(p_{e}^{h}\right)^{*}=\frac{1}{4}\left(\tilde{p}_{\varepsilon e}^{1}-\tilde{p}_{\varepsilon e}^{2}+\tilde{p}_{\varepsilon e}^{3}-\tilde{p}_{\varepsilon e}^{4}\right)
$$

$$
\tilde{p}_{\varepsilon e}^{i}=-\frac{1}{\varepsilon} \operatorname{div} \boldsymbol{u}_{\varepsilon}^{h}\left(\xi_{e}^{i}\right), \quad 1 \leqslant i \leqslant 4 .
$$

Now define

$$
\begin{array}{ll}
p_{\varepsilon e}^{1}=\tilde{p}_{\varepsilon e}^{1}-\left(p_{e}^{h}\right)^{*}, & p_{\varepsilon e}^{2}=\tilde{p}_{\varepsilon e}^{2}+\left(p_{e}^{h}\right)^{*}, \\
p_{\varepsilon e}^{3}=\tilde{p}_{\varepsilon e}^{3}-\left(p_{e}^{h}\right)^{*}, & p_{\varepsilon e}^{4}=\tilde{p}_{\varepsilon e}^{4}+\left(p_{e}^{h}\right)^{*} .
\end{array}
$$

Then it is clear that

$$
p_{\varepsilon}^{h} \in Q_{h},
$$

that is, the adjusted pressure $p_{\varepsilon}^{h}$ by (8.9) belongs to the space $Q_{h}$ defined as (8.4). Furthermore, within a composite element $\Omega_{e}$, the identity

$$
I_{e}\left(\tilde{p}_{\varepsilon}^{h}, \operatorname{div} v^{h}\right)=I_{e}\left(p_{\varepsilon}^{h}, \operatorname{div} v^{h}\right), \quad \forall v^{h} \in V_{h} \text { such that } v^{h}=0 \text { on } \partial \Omega_{e}
$$


holds for $p_{\varepsilon}^{h}$ defined by (8.8). That is

$$
\frac{1}{\varepsilon} I\left(\operatorname{div} \boldsymbol{u}_{\varepsilon}^{h}, \operatorname{div} \boldsymbol{v}^{h}\right)=-\left(p_{\varepsilon}^{h}, \operatorname{div} \boldsymbol{v}^{h}\right)
$$

is satisfied, as is required. Thus the condition (2) in Section 4 must now be changed according to:

$\left(2^{\prime}\right) \quad$ There exists an element $p_{\varepsilon}^{h} \in Q_{h}$ defined by (8.8).

Now we shall verify that condition (3) in Section 4, the discrete LBB-condition, holds on the special space $Q_{h}$ defined by (8.4). To do this, we first record some properties of finite element approximations following $[7,8]$.

\subsection{Some preliminaries}

For simplicity, we assume that $\Omega$ is a polygonal domain. Let $T_{h}$ be a triangulation of $\bar{\Omega}$ composed of $E$ rectangles $\Omega_{e}$ with diameters bounded by $h_{e}$ such that

$$
\bar{\Omega}=\bigcup_{e=1}^{E} \bar{\Omega}_{e} .
$$

Each rectangle $\bar{\Omega}_{e}$ is characterized by two numbers

- $h_{e}=$ diameter of $\Omega_{e}$; and

- $\rho_{e}=$ diameter of the largest circle contained in $\Omega_{e}$.

For simplicity, we will confine our attention to refinements in which there exists a number $\sigma>0$ such that $h_{e} / \rho_{e}=\sigma_{e} \leqslant \sigma, 1 \leqslant e \leqslant E$.

We denote by $\hat{\Omega}$ a reference rectangle and by $\hat{\boldsymbol{x}}=\left(\hat{x}_{1}, \hat{x}_{2}\right)$ the cartesian coordinates of points in $\hat{\Omega}$. The elements $\Omega_{e} \subset \mathscr{T}_{h}$ are affine equivalent to $\hat{\Omega}$ in the sense that there exists affine envertible map $F_{e}$ such that

$$
F_{e}(\hat{\boldsymbol{x}})=B_{e}(\hat{\boldsymbol{x}})+b=x \in \bar{\Omega}_{e} .
$$

For each function $v_{e}$ defined on $\bar{\Omega}_{e}$ we associate a function $\hat{v}$ defined over $\hat{\Omega}$ by

$$
\hat{v}(\hat{\boldsymbol{x}})=v_{e}\left(F_{e}(\hat{\boldsymbol{x}})\right), \quad \hat{\boldsymbol{x}} \in \Omega .
$$

Likewise, if $\Pi \in \mathscr{L}\left(H^{k+1}\left(\Omega_{e}\right), H^{m}\left(\Omega_{e}\right)\right), \quad k+1>m>0, \quad$ we define an operator $\hat{\Pi} \in$ $\mathscr{L}\left(H^{k+1}(\hat{\Omega}), H^{m}(\hat{\Omega})\right)$ by

$$
\hat{\Pi} \hat{v}=\widehat{\Pi v}, \quad \forall v \in H^{k+1}(\Omega) .
$$

Also, the matrices $B_{e}$ in $(8.15)$ have the property that

$$
\left\|B_{e}\right\| \leqslant h_{e} / \hat{\rho}, \quad\left\|B_{e}^{-1}\right\| \leqslant \hat{h} / \rho_{e}
$$


where $\|\cdot\|$ denotes the matrix norm, $\hat{h}=\operatorname{diag}(\hat{\Omega})$, and $\hat{\rho}$ is the diameter of the largest sphere contained in $\hat{\Omega}$.

All of the above properties are developed in full in the book of Ciarlet [7]. In addition, we will need the following properties of such finite element families proved in [7].

LEMMA 8.1. For $m \geqslant 0$, the map $v_{e} \rightarrow \hat{v}=v_{e} \circ F_{e}$ is an isomorphism from $H^{m}\left(\Omega_{e}\right)$ onto $H^{m}(\hat{\Omega})$. Moreover, constants $C_{1}, C_{2}$ exist, depending only on $\hat{\Omega}$ and $m$, such that

$$
|\hat{v}|_{m, \Omega} \leqslant C_{1}|| B_{e}\left\|^{m}\left|\operatorname{det} B_{e}\right|^{1 / 2}|v|_{m, \Omega_{e}}, \quad|v|_{m, \Omega_{e}} \leqslant C_{2}\right\| B_{e}^{-1} \|^{m}\left|\operatorname{det} B_{e}\right|^{-1 / 2}|\hat{v}|_{m, \Omega}
$$

where $|\cdot|_{m, \Omega}$ is the seminorm,

$$
|\hat{v}|_{m, \hat{\Lambda}}=\left\{\int_{\hat{\Omega}} \sum_{|\alpha|=m}\left|D^{\alpha} \hat{v}\right|^{2} \mathrm{~d} \hat{x}\right\}^{1 / 2}
$$

We also need the companion lemma.

LEMMA 8.2. Let $k$ and $m$ be integers such that $0 \leqslant m \leqslant k+1$ and let $\Pi_{e} \in$ $\mathscr{L}\left(H^{k+1}\left(\Omega_{e}\right), H^{m}\left(\Omega_{e}\right)\right)$ be defined by

$$
\left(\Pi_{e} v_{e}\right) \circ F_{e}=\hat{\Pi}\left(v \circ F_{e}\right)
$$

where $\hat{\Pi} \in \mathscr{L}\left(H^{k+1}(\hat{\Omega}), H^{m}(\hat{\Omega})\right)$ is a projection operator that preserves polynomials of degree $\leqslant k ;$ i.e., if $P_{k}(\hat{\Omega})$ is the space of polynomials of degree $\leqslant k$ on $\hat{\Omega}$, then

$$
\hat{\Pi} p=p, \quad \forall p \in P_{k}(\hat{\Omega}) .
$$

Then there exists a constant $C$, independent of $v_{e}$ and $\Omega_{e}$, such that

$$
\left|v_{e}-\Pi_{e} v_{e}\right|_{m, \Omega_{e}} \leqslant C\left\|B_{e}\right\|^{k+1}\left\|B_{e}^{-1}\right\|^{m}\left|v_{e}\right|_{k+1, \Omega_{e}}, \quad \forall v_{e} \in H^{k+1}\left(\Omega_{e}\right) .
$$

\subsection{Discrete LBB-condition}

We shall now show the existence of an element $\boldsymbol{w}^{h} \in V_{h}$ satisfying (7.1) for a given $q^{h} \in Q_{h}$.

LEMMA 8.3. For the finite-dimensional spaces $V_{h}$ and $Q_{h}$ defined by (8.2) and (8.4), there exists an element $v^{h} \in V_{h}$ satisfying the following condition.

$$
\text { For any } v \in\left(H^{1}(\Omega)\right)^{2}, \quad\left(q^{h}, \operatorname{div}\left(v^{h}-v\right)\right)=0, \forall q^{h} \in Q_{h} .
$$

PROOF. It suffices to prove (8.23) on a typical composite element $\Omega_{e}$; i.e., we shall show that

$$
\left(q^{h}, \operatorname{div} v^{h}\right)_{\Omega_{e}}=\left(q^{h}, \operatorname{div} v\right)_{\Omega_{e}}, \quad 1 \leqslant e \leqslant E
$$

or, equivalently (since $\operatorname{grad} q_{e}^{h}=0$ ), 


$$
\sum_{i=1}^{4} \int_{\partial K_{e}^{i}} q^{h} v^{h} \cdot n \mathrm{~d} s=\sum_{i=1}^{4} \int_{\partial K_{e}^{i}} q^{h} \boldsymbol{v} \cdot n \mathrm{~d} s
$$

where $n$ is outward unit normal on the boundaries of subrectangle $K_{e}^{i}$. Using the notations in Fig. 5, let $\left\{\phi_{j}\right\}_{j=1}^{9}$ denote the piecewise bilinear basis functions defining $v^{h}$ so that

$$
\begin{aligned}
v^{h}(x) & =(\tilde{U}(x), \tilde{V}(x)), \\
\tilde{U}(x) & =\sum_{j=1}^{9} \tilde{U}_{j} \phi_{j}(x), \quad \tilde{V}(x)=\sum_{j=1}^{9} \tilde{V}_{j} \phi_{j}(x), \\
\phi_{j}\left(a_{i}\right) & =\delta_{j i}, \quad 1 \leqslant i, j \leqslant 9 .
\end{aligned}
$$

Then by a direct expansion of (8.25) we construct the following set of equations:

$$
\begin{aligned}
& \left(q_{1}+q_{2}\right) \tilde{V}_{6}=\left(q_{1}+q_{2}\right)\left\{\frac{1}{2 h} \int_{1}^{2} v \mathrm{~d} x_{1}-\frac{1}{2}\left(\tilde{V}_{1}+\tilde{V}_{2}\right)\right\}, \\
& \left(q_{3}+q_{1}\right) \tilde{V}_{8}=\left(q_{3}+q_{4}\right)\left\{\frac{1}{2 h} \int_{4}^{3} v \mathrm{~d} x_{1}-\frac{1}{2}\left(\tilde{V}_{3}+\tilde{V}_{4}\right)\right\}, \\
& \left(q_{2}+q_{3}\right) \tilde{U}_{7}=\left(q_{2}+q_{3}\right)\left\{\frac{1}{2 h} \int_{2}^{3} u \mathrm{~d} x_{2}-\frac{1}{2}\left(\tilde{U}_{2}+\tilde{U}_{3}\right)\right\}, \\
& \left(q_{1}+q_{4}\right) \tilde{U}_{9}=\left(q_{1}+q_{4}\right)\left\{\frac{1}{2 h} \int_{1}^{4} u \mathrm{~d} x_{2}-\frac{1}{2}\left(\tilde{U}_{1}+\tilde{U}_{4}\right)\right\},
\end{aligned}
$$

where $v=(u, v)$ and $h$ is the length of a side of $K_{e}^{i}$. Also,

$$
\begin{aligned}
& \left(q_{1}-q_{2}-q_{3}+q_{4}\right) \tilde{U}_{5}= \\
& =\left(q_{1}-q_{2}\right)\left\{\frac{1}{2}\left(\frac{1}{h} \int_{0}^{5} u \mathrm{~d} x_{2}-\tilde{U}_{6}\right)+\frac{1}{4}\left(\frac{1}{h} \int_{6}^{2} v \mathrm{~d} x_{1}-\frac{1}{h} \int_{1}^{6} v \mathrm{~d} x_{1}\right)+\frac{1}{4}\left(\tilde{V}_{1}-\tilde{V}_{2}\right)\right\} \\
& \quad+\left(q_{4}-q_{3}\right)\left\{\frac{1}{2}\left(\frac{1}{h} \int_{5}^{8} u \mathrm{~d} x_{2}-\tilde{U}_{8}\right)+\frac{1}{4}\left(\frac{1}{h} \int_{4}^{8} v \mathrm{~d} x_{1}-\frac{1}{h} \int_{8}^{3} v \mathrm{~d} x_{1}\right)+\frac{1}{4}\left(\tilde{V}_{3}-\tilde{V}_{4}\right)\right\} \\
& \left(q_{1}+q_{2}-q_{3}-q_{4}\right) \tilde{V}_{5}= \\
& =\left(q_{1}-q_{4}\right)\left\{\frac{1}{2}\left(\frac{1}{h} \int_{9}^{5} v \mathrm{~d} x_{1}-\tilde{V}_{9}+\frac{1}{4}\left(\frac{1}{h} \int_{9}^{4} u \mathrm{~d} x_{2}-\frac{1}{h} \int_{1}^{9} u \mathrm{~d} x_{2}\right)+\frac{1}{4}\left(U_{1}-U_{4}\right)\right\}\right. \\
& \left.\quad+\left(q_{2}-q_{3}\right)\left\{\frac{1}{2}\left(\frac{1}{h} \int_{5}^{7} v \mathrm{~d} x_{1}-\tilde{V}_{7}\right)+\frac{1}{h} \int_{2}^{7} u \mathrm{~d} x_{2}-\frac{1}{h} \int_{7}^{3} u \mathrm{~d} x_{2}\right)+\frac{1}{4}\left(\tilde{U}_{3}-\tilde{U}_{2}\right)\right\}
\end{aligned}
$$

Hence, using (8.5), (8.27) and (8.28) yicld 


$$
\begin{aligned}
\tilde{V}_{6}= & \frac{1}{2 h} \int_{1}^{2} v \mathrm{~d} x_{1}-\frac{1}{2}\left(\tilde{V}_{1}-\tilde{V}_{2}\right), \quad \tilde{U}_{7}=\frac{1}{2 h} \int_{2}^{3} u \mathrm{~d} x_{2}-\frac{1}{2}\left(\tilde{U}_{2}+\tilde{U}_{3}\right) \\
\tilde{V}_{8}= & \frac{1}{2 h} \int_{4}^{3} v \mathrm{~d} x_{1}-\frac{1}{2}\left(\tilde{V}_{3}+\tilde{V}_{4}\right), \quad \tilde{U}_{9}=\frac{1}{2 h} \int_{1}^{4} u \mathrm{~d} x_{2}-\frac{1}{2}\left(\tilde{U}_{1}+\tilde{U}_{4}\right) \\
\tilde{U}_{5}= & \frac{1}{2}\left\{\frac{1}{h} \int_{6}^{8} u \mathrm{~d} x_{2}-\left(\tilde{U}_{6}+\tilde{U}_{8}\right)\right\} \\
& +\frac{1}{4}\left\{\left(-\frac{1}{h} \int_{1}^{6} v \mathrm{~d} x_{1}+\tilde{V}_{1}\right)+\left(\frac{1}{h} \int_{6}^{2} v \mathrm{~d} x_{1}-\tilde{V}_{2}\right)\right. \\
& \left.+\left(-\frac{1}{h} \int_{8}^{3} v \mathrm{~d} x_{1}+\tilde{V}_{3}\right)+\left(\frac{1}{h} \int_{4}^{8} v \mathrm{~d} x_{1}-\tilde{V}_{4}\right)\right\} \\
\tilde{V}_{5}= & \frac{1}{2}\left\{\frac{1}{h} \int_{9}^{7} v \mathrm{~d} x_{1}-\left(\tilde{V}_{7}+\tilde{V}_{9}\right)\right\} \\
& +\frac{1}{4}\left\{\left(-\frac{1}{h} \int_{1}^{9} u \mathrm{~d} x_{2}+\tilde{U}_{1}\right)+\left(\frac{1}{h} \int_{2}^{7} u \mathrm{~d} x_{2}-\tilde{U}_{2}\right)\right. \\
& \left.+\left(-\frac{1}{h} \int_{7}^{3} u \mathrm{~d} x_{2}+\tilde{U}_{3}\right)+\left(\frac{1}{h} \int_{9}^{4} u \mathrm{~d} x_{2}-\tilde{U}_{4}\right)\right\}
\end{aligned}
$$

In the composite element $\Omega_{3}, v^{h}$ has 18 degrees of freedom. Eq. (8.29) represents six independent relationships. Once the 12 components of $v^{h}$ appearing on the right-hand side of (8.29) are specified, we could determine the values of the 12 components of the right-hand side of ( 8.29$)$ by equating the nodal values of $v$ and $v^{h}$. Since $v$ may not be smooth enough to allow this computation, we will use instead of $v$ its $H^{1}$ projection onto $V_{h}$. Specifically, let $w^{h}$ be the unique vector defined by

$$
\left(\nabla\left(v-w^{h}\right), \nabla z^{h}\right)=0, \quad \forall z^{h} \in V_{h}
$$

We denote $w^{h}=\left(\sum_{i=1}^{9} \bar{U}_{i} \phi_{i}, \sum_{i=1}^{9} \bar{V}_{i} \phi_{i}\right)$, and choose the remaining 12 degrees of freedom of $v^{h}$ as follows.

$$
\begin{array}{ll}
\tilde{U}_{i}=\bar{U}_{i}, & i=1,2,3,4,6,8 \\
\tilde{V}_{i}=\bar{V}_{i}, & i=1,2,3,4,7,9 .
\end{array}
$$

Here index $i$ indicates the nodal numbering in $\Omega_{e}$ as shown in Fig. 5.

We have thus shown that for every $v \in\left(H^{1}(\Omega)\right)^{2}$ there is a unique $v^{h} \in V_{h}$ satisfying (8.23).

LEMMA 8.4. There exists a constant $C>0$, independent of $h$, such that

$$
\left\|v^{h}\right\|_{1} \leqslant C\|v\|_{1}
$$

for the element $v^{h}$ satisfying (8.23). 
PROOF. We first note that (8.29) implies that

where

$$
\int_{i}^{i} e \cdot n \mathrm{~d} s=0, \quad 1 \leqslant i \leqslant j \leqslant 4
$$

$$
\boldsymbol{e}=\boldsymbol{v}-\boldsymbol{v}^{h}
$$

In addition, along the sides of the subrectangles which pass through the center node of $\Omega_{e}$,

$$
\begin{aligned}
& \int_{6}^{8} e \cdot i_{1} \mathrm{~d} x_{2}-\frac{1}{2}\left\{\int_{1}^{6} e \cdot i_{2} \mathrm{~d} x_{1}-\int_{6}^{2} e \cdot i_{2} \mathrm{~d} x_{1}+\int_{8}^{3} e \cdot i_{2} \mathrm{~d} x_{1}-\int_{4}^{8} e \cdot i_{2} \mathrm{~d} x_{1}\right\}=0, \\
& \int_{9}^{7} e \cdot i_{2} \mathrm{~d} x_{1}-\frac{1}{2}\left\{\int_{1}^{9} e \cdot i_{1} \mathrm{~d} x_{2}-\int_{9}^{4} e \cdot i_{1} \mathrm{~d} x_{2}+\int_{7}^{3} e \cdot i_{1} \mathrm{~d} x_{2}-\int_{2}^{7} e \cdot i_{1} \mathrm{~d} x_{2}\right\}=0 .
\end{aligned}
$$

Here $i_{1}$ and $i_{2}$ are unit bases vectors directed along $x_{1}$ and $x_{2}$, respectively.

Next, we define the error functions

$$
\boldsymbol{E}_{h}=\boldsymbol{v}^{h}-\boldsymbol{w}^{h} \in V_{h} ; \quad \boldsymbol{\xi}=\boldsymbol{v}-\boldsymbol{w}^{h}
$$

where $\boldsymbol{w}^{h}$ is the $\boldsymbol{H}^{1}(\Omega)$-projection of $v$ into $V_{h}$ (introduced in the proof of Lemma 8.3). Thus,

$$
\left|\boldsymbol{v}^{h}\right|_{1, \Omega} \leqslant\left|\boldsymbol{w}^{h}\right|_{1, \Omega}+\left|\boldsymbol{E}_{h}\right|_{1, \Omega} \leqslant|\boldsymbol{v}|_{\imath, \Omega}+\left|\boldsymbol{E}_{h}\right|_{1, \Omega} .
$$

Thus, we need to obtain an estimate of $\left|\boldsymbol{E}_{h}\right|_{1, \Omega}$.

Toward this end, we note that $\boldsymbol{E}_{h}\left(\boldsymbol{a}_{i}\right)=\mathbf{0}, i=1,2,3,4$ and that along the sides $\overline{12}, \overline{23}, \overline{34}$ and $\overline{41}, \boldsymbol{E}_{h} \cdot \boldsymbol{t}=0$ where $\boldsymbol{t}$ is the unit vector tangent to each of these sides. Also, since $\boldsymbol{e}=\boldsymbol{E}_{\boldsymbol{h}}-\boldsymbol{\xi}$, $(8.33 \mathrm{a})$ and $(8.33 \mathrm{c})$ yield the conditions

$$
\begin{aligned}
& \int_{i}^{i}\left(\boldsymbol{E}_{h}-\xi\right) \cdot n \mathrm{~d} s=0, \quad 1 \leqslant i \leqslant j \leqslant 4, \\
& \int_{6}^{8}\left(\boldsymbol{E}_{h}-\xi\right) \cdot \boldsymbol{i}_{1} \mathrm{~d} x_{2}-\frac{1}{2} \int_{1}^{6} \xi \cdot \boldsymbol{i}_{2} \mathrm{~d} x_{1} \\
& \quad-\int_{6}^{2} \xi \cdot \boldsymbol{i}_{2} \mathrm{~d} x_{1}+\int_{8}^{3} \xi \cdot \boldsymbol{i}_{2} \mathrm{~d} x_{1}-\int_{4}^{5} \xi \cdot \boldsymbol{i}_{2} \mathrm{~d} x_{1}=0, \\
& \int_{9}^{7}\left(\boldsymbol{E}_{h}-\xi\right) \cdot \boldsymbol{i}_{2} \mathrm{~d} x_{1}+\frac{1}{2} \int_{1}^{9} \xi \cdot i_{1} \mathrm{~d} x_{2} \\
& \quad-\int_{9}^{4} \xi \cdot i_{1} \mathrm{~d} x_{2}+\int_{7}^{3} \xi \cdot i_{1} \mathrm{~d} x_{2}-\int_{2}^{7} \xi \cdot i_{1} \mathrm{~d} x_{2}=0 .
\end{aligned}
$$

In these last two equalities, we have used the fact that $\boldsymbol{E}_{h}\left(\boldsymbol{a}_{i}\right)=0, i=1,2,3,4$ and that the subrectangles have equal dimensions.

In each composite element, $\boldsymbol{E}_{h}$ can be expressed in the form 


$$
E_{h}(x)=E_{h}\left(a_{5}\right) \phi_{5}\left(a_{5}\right)+\sum_{i \leqslant i \leqslant j \leqslant 4} E_{h}\left(a_{i j}\right) \phi_{i j}(x)
$$

where $\boldsymbol{a}_{5}$ is the center node, $\boldsymbol{a}_{i j}$ the midside nodes on side $\left[\boldsymbol{a}_{i}, \boldsymbol{a}_{i}\right]$, and $\boldsymbol{\phi}_{5}, \boldsymbol{\phi}_{i j}$ the corresponding local, piecewise bilinear shape functions for the element. Only six of the 18 nodal values of $\boldsymbol{E}_{h}$ are nonzero and can be determined by conditions (8.36). To use these conditions, we note that for continuous $f$,

$$
\int_{\Omega_{e}} f \mathrm{~d} x=\left|\operatorname{det} B_{e}\right| \int_{\hat{\Omega}} \hat{f} \mathrm{~d} \hat{x} .
$$

Hence, by integrating $\boldsymbol{E}_{h}$ along side $\left[\boldsymbol{a}_{i}, \boldsymbol{a}_{j}\right]$ of $\Omega_{e}$, we have

$$
\boldsymbol{E}_{h}\left(\boldsymbol{a}_{i j}\right)=\left[\int_{i}^{j} \hat{\boldsymbol{\phi}}_{i j} \mathrm{~d} \hat{\boldsymbol{s}}\right]^{-1} \int_{i}^{j} \hat{\boldsymbol{\xi}} \mathrm{d} \hat{s}
$$

$\left(\hat{\boldsymbol{\xi}}=\boldsymbol{\xi} \circ \boldsymbol{F}_{e}\right)$. Also, for the components of $\boldsymbol{E}_{\boldsymbol{h}}$ at the center node $\boldsymbol{a}_{5}$, we have

$$
\begin{gathered}
\boldsymbol{E}_{h}\left(\boldsymbol{a}_{5}\right) \cdot \boldsymbol{i}_{1}=\left[\int_{6}^{8} \hat{\boldsymbol{\phi}}_{5} \mathrm{~d} \hat{x}_{2}\right]^{-1}\left\{\int_{6}^{8} \hat{\xi} 1 \mathrm{~d} \hat{x}_{2}+\frac{1}{2}\left[\int_{1}^{2} \hat{\xi}^{2} \mathrm{~d} \hat{x}_{1}\right.\right. \\
\left.\left.+\int_{4}^{3} \hat{\xi}^{2} \mathrm{~d} \hat{x}_{1}-\int_{6}^{2} \hat{\xi}^{2} \mathrm{~d} x_{1}-\int_{4}^{8} \hat{\xi}^{2} \mathrm{~d} \hat{x}_{1}\right]\right\} \\
\boldsymbol{E}_{h}\left(\boldsymbol{a}_{5}\right) \cdot \boldsymbol{i}_{2}=\left[\int_{9}^{7} \hat{\boldsymbol{\phi}}_{5} \mathrm{~d} \hat{x}_{1}\right]^{-1}\left\{\int_{9}^{7} \hat{\xi}^{2} \mathrm{~d} \hat{x}_{1}+\frac{1}{2}\left[\int_{9}^{4} \hat{\xi}^{1} \mathrm{~d} \hat{x}_{2}\right.\right. \\
\left.\left.+\int_{2}^{7} \hat{\xi}^{1} \mathrm{~d} \hat{x}_{2}-\int_{1}^{9} \hat{\xi}^{1} \mathrm{~d} \hat{x}_{2}-\int_{7}^{3} \hat{\xi}^{1} \mathrm{~d} \hat{x}_{2}\right]\right\}
\end{gathered}
$$

Hence, (8.38) and (8.39) lead us to the inequalities,

$$
\left|\boldsymbol{E}_{h}\left(\boldsymbol{a}_{i}\right)\right|=\left|\boldsymbol{E}_{h}\left(\boldsymbol{a}_{l}\right)\right| \leqslant C\|\hat{\boldsymbol{\xi}}\|_{0, \partial \Omega} \leqslant C\left\{\|\hat{\xi}\|_{0, \Omega}^{2}+|\hat{\boldsymbol{\xi}}|_{1, \Omega}^{2}\right\}^{1 / 2}
$$

where $i=1, \ldots, 9, k=5, \ldots, 9$ and $|\cdot|$ denotes the Eucledian norm in $\mathbb{R}^{2}$. We have from (8.19)

$$
\left|\boldsymbol{E}_{h}\left(\boldsymbol{a}_{i}\right)\right| \leqslant C\left|\operatorname{det} B_{e}\right|^{-1 / 2}\left(\|\xi\|_{0, \Omega_{e}}^{2}+\left\|B_{e}\right\|^{2}|\xi|_{1, \Omega_{e}}^{2}\right)^{1 / 2}
$$

On the other hand, (8.19) also shows that

$$
\left|\phi_{i}\right|_{1, \Omega_{e}} \leqslant C\left\|B_{e}^{-1}\right\|\left|\operatorname{det} B_{e}\right|^{1 / 2}\left|\hat{\phi}_{i}\right|_{1, \Omega} \leqslant \hat{C}\left\|B_{e}^{-1}\right\|\left|\operatorname{det} B_{e}\right|^{1 / 2} .
$$

Combining (8.41) and (8.19) gives

$$
\begin{aligned}
\left|\boldsymbol{E}_{h}\right|_{1, \Omega_{e}} & \leqslant \sum_{i=5}^{9}\left|\boldsymbol{E}_{h}\left(\boldsymbol{a}_{i}\right) \| \phi_{i}\right|_{1, \Omega_{e}} \\
& \leqslant C\left\|B_{e}^{-1}\right\|\left(\|\xi\|_{0, \Omega_{e}}^{2}+\left\|B_{e}\right\|^{2}|\xi|_{1, \Omega_{e}}^{2}\right)^{1 / 2} .
\end{aligned}
$$


Thus, according to $(8.18)$

Thus,

$$
\left|\boldsymbol{E}_{h}\right|_{1, \Omega_{e}} \leqslant C \sigma\left\{h_{e}^{-2}\|\xi\|_{0, \Omega_{e}}^{2}+|\boldsymbol{\xi}|_{1, \Omega_{e}}^{2}\right\}^{1 / 2} .
$$

$$
\left|\boldsymbol{E}_{h}\right|_{1, \Omega} \leqslant C\left\{h^{-2}\|\boldsymbol{\xi}\|_{0, \Omega}^{2}+|\boldsymbol{\xi}|_{1, \Omega}^{2}\right\}^{1 / 2} .
$$

Our problem thus reduces to one of obtaining an estimate of $\|\boldsymbol{\xi}\|_{0, \Omega}$ and $|\boldsymbol{\xi}|_{1, \Omega}\left(\boldsymbol{\xi}=\boldsymbol{v}-\boldsymbol{w}^{h}\right)$ in terms of $|v|_{1}$. We immediately have

$$
|\boldsymbol{\xi}|_{1, \Omega} \leqslant|\boldsymbol{v}|_{1}
$$

because $\boldsymbol{w}^{h}$ is, by definitions, the $\boldsymbol{H}_{0}^{1}$-orthogonal projection of $\boldsymbol{v}$. Thus, we need only estimate $|\xi|_{0, \Omega}$. This can be accomplished by the duality agreement of the well-known Aubin-Nitsche method.

We first consider the auxiliary problem,

$$
-\Delta z=g \text { in } \Omega,\left.\quad z\right|_{\Gamma}=0 \text { on } \Gamma .
$$

For which it is known that a constant $C$ exists such that $\|z\|_{2, \Omega} \leqslant C\|g\|_{0, \Omega}$. Thus, for a given $g \in L^{2}(\Omega)$

$$
\left(\xi^{i}, g\right)=-\left(\xi^{i}, \Delta z\right)=\int_{\Omega} \nabla \xi^{i} \cdot \nabla z \mathrm{~d} x, \quad i=1,2
$$

and, again using the orthogonality of the error,

$$
\left(\xi^{i}, g\right)=\left(\nabla \xi^{i}, \nabla\left(z-z^{h}\right)\right) \leqslant\left|\xi^{i}\right|_{1, \Omega}\left|z-z^{h}\right|_{1, \Omega}, \quad \forall z^{h} \in S_{h}
$$

where $V_{h}=\left(S_{h}\right)^{2}$. Choosing $z^{h}$ to be the interpolant in $S_{h}$ of $z$, we have (from (8.22) with $k=m=1$ ),

$$
\left(\xi^{i}, g\right) \leqslant\left|\xi^{i}\right|_{1, \Omega}\left(C h\|z\|_{2, \Omega}\right) \leqslant C h\left|\xi^{i}\right|_{1, \Omega}\|g\|_{0, \Omega},
$$

so that

Hence,

$$
\left\|\xi^{i}\right\|_{0, \Omega}=\sup _{g \in L^{2}(\Omega)} \frac{\left(\xi^{i}, g\right)}{\|g\|_{0, \Omega}} \leqslant C h\left|\xi^{i}\right|_{1, \Omega}, \quad i=1,2 .
$$

$$
\|\boldsymbol{\xi}\|_{1, \Omega} \leqslant C h|\xi|_{1, \Omega} \leqslant C h|v|_{1, \Omega} .
$$

Collecting (8.35), (8.44), (8.45) and (8.46), we have

$$
\begin{aligned}
\left\|\boldsymbol{v}^{h}\right\|_{1, \Omega} & \leqslant|\boldsymbol{v}|_{1, \Omega}+C\left\{h^{-2}\|\boldsymbol{\xi}\|_{0, \Omega}^{2}+|\boldsymbol{\xi}|_{1, \Omega}^{2}\right\}^{1 / 2} \\
& \leqslant|\boldsymbol{v}|_{1, \Omega}+C\left\{h^{-2}\left(C h|\boldsymbol{v}|_{1, \Omega}\right)^{2}+|\boldsymbol{v}|_{1, \Omega}^{2}\right\}^{1 / 2} \\
& \leqslant C|\boldsymbol{v}|_{1, \Omega}
\end{aligned}
$$

as asserted. 
Using Lemmas 8.3 and 8.4 , we have:

THEOREM 8.5. Under conditions (P.1) and (P.2) on $\Omega$, on refinements of the mesh, and the spaces $V_{h}$ and $Q_{h}$ listed earlier, there exists a constant $\alpha$, independent of $h$, such that

$$
\alpha\left\|q^{h}\right\|_{0} \leqslant \sup _{v^{h} \in V_{h}} \frac{\left(q^{h}, \operatorname{div} v^{h}\right)}{\left\|v^{h}\right\|_{1}}, \forall q^{h} \in Q_{h}
$$

where $V_{h}$ is defined in (8.2) and $Q_{h}$ in (8.4).

Thus, we can conclude the convergence of the penalty method (4.2) for $Q_{1}$-elements with the '1-point' Gaussian integration rule under the assumptions that the domain $\Omega$ is a rectangle, and that the domain is uniformly discretized by equal size subrectangles. That is:

THEOREM 8.6. Let conditions (P.1) and (P.2) listed earlier (particularly, (8.1)-(8.4)) and condition $\left(2^{\prime}\right)$ hold. Let $\left(u^{h}, p^{h}\right) \in V_{h} \times Q_{h}$ be the mixed finite element approximation of (2.1) obtained as the limit, as $\varepsilon \rightarrow 0$, of the solutions $\left(\boldsymbol{u}_{\varepsilon}^{h}, p_{\varepsilon}^{h}\right)$ of the penalty-approximation obtained using the spaces and projection methods described earlier. Finally, let the solution to (7.1) satisfy

$$
(\boldsymbol{u}, p) \in \boldsymbol{H}^{2}(\Omega) \times H^{1}(\Omega) .
$$

Then

$$
\left\|\boldsymbol{u}-\boldsymbol{u}^{h}\right\|_{1}+\left\|p-p^{h}\right\|_{0} \leqslant C\left(\|\boldsymbol{u}\|_{2}+\|p\|_{1}\right) h
$$

where $C$ is a positive constant independent of $h$.

\section{Numerical example}

A typical example problem is described which is designed to verify some of the results obtained thus far. This example involves the Dirichlet problem for Stokesian flow in which the fluid is subjected to a constant body force $f=(800,800)$ applied over a square subdomain $\Omega_{0}$, as shown in Fig. 6 . We take $\mu=333$ and the penalty parameter $\varepsilon=10^{-5}$. We use a rather coarse mesh of 16 elements and choose

$$
\begin{aligned}
& Q_{2} \text {-elements (9-node biquadratics) for } V_{h}, \\
& I() \sim 2 \times 2 \text {-Gaussian quadrature, }
\end{aligned}
$$

Fig. 6 shows computed hydrostatic pressures which seem to be smoothly distributed along sections $A^{\prime}-A^{\prime}$. The method (9.1) is stable for this case, and the results appear to be quite satisfactory.

For the choice 
A

- A : 9-node 1-point Gaussian quadrature

$A^{\prime}--0-A^{\prime}:$ 9-node $2 \times 2-p t$. Gaussian quadrature
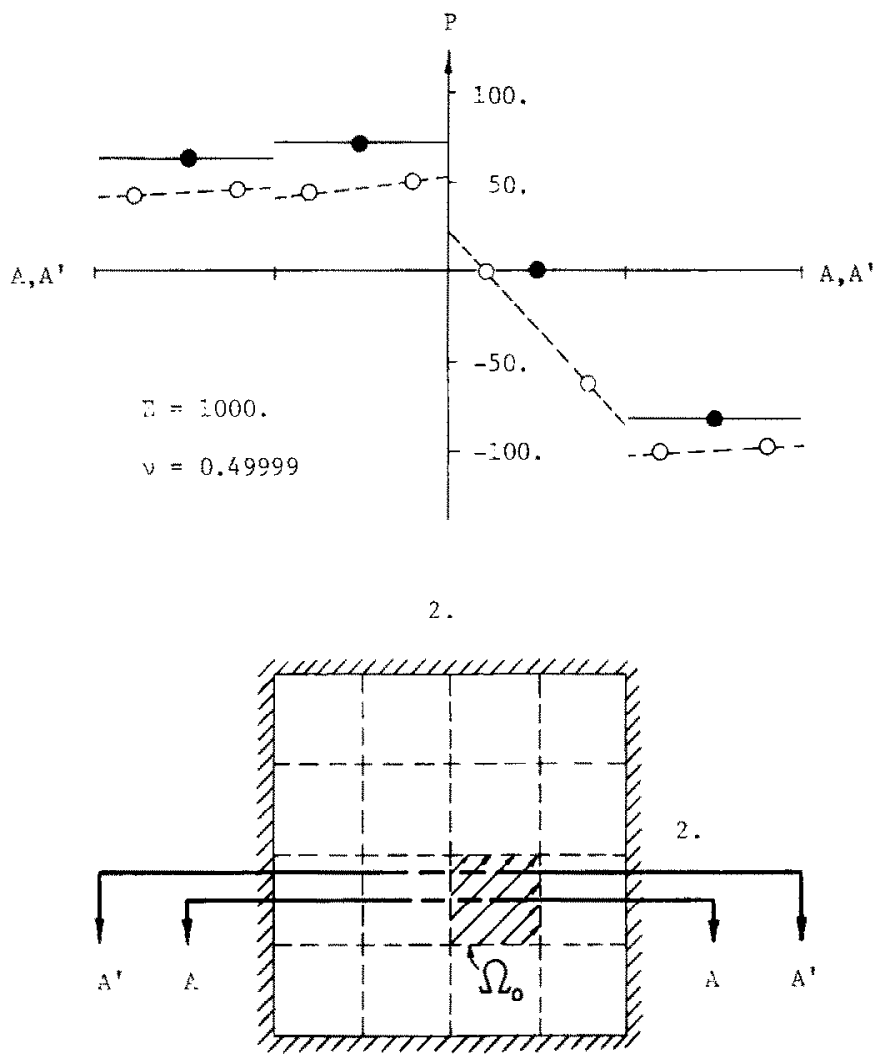

Fig. 6. Pressure distribution across the sections $A-A$ and $A^{\prime}-A^{\prime}$ of the example in Section 9 (uniform load).

$Q_{2}$-elements (9-node biquadratics) for $V_{h}$, $I$ () 1-point Gaussian quadrature,

again a smooth pressure distribution is obtained as is also shown in Fig. 6.

For other choices of data, we observe one major difference between methods (9.1) and (9.2): method (9.2) appears to be quite robust and insensitive to singularities whereas method (9.1) behaved well only so long as the data were smooth. In particular, if a point load $f=200(\delta(x-\bar{x}, y), \delta(x, y-\bar{y}))$ is applied at point $(\bar{x}, \bar{y}) \in \Omega$, then similar oscillation to the checkerboard modes in ker $B_{h}^{*}$ appear to be activated, and we obtain pressures in which such modes are superimposed upon those obtained using method (9.2). It is significant that the LBB-conditions (4.9) hold for method (9.2) with $\alpha_{h}$ independent of $h$ whereas the unstable method $(9.1), \alpha_{h}=\mathrm{O}\left(h^{2}\right)$. 

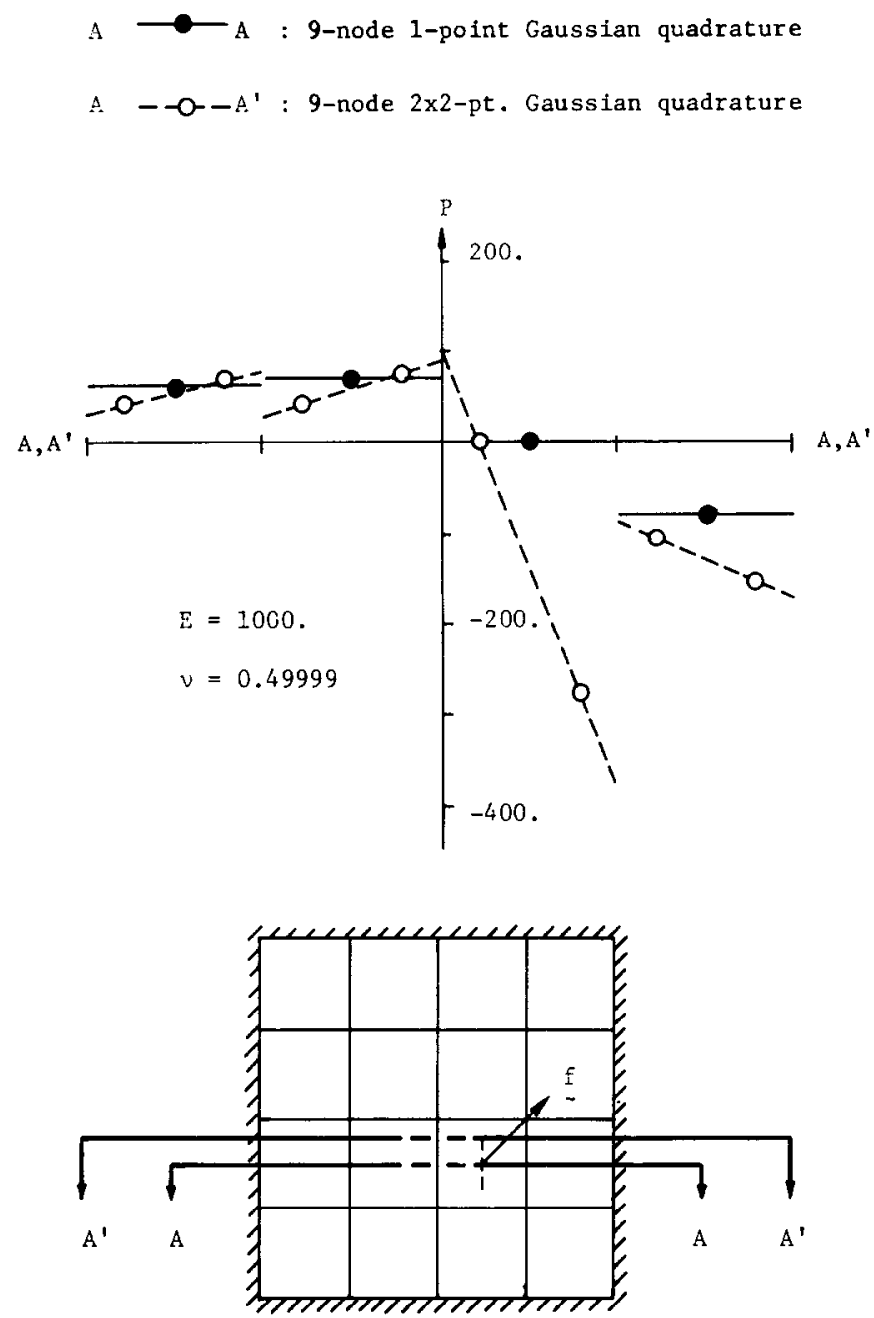

Fig. 7. Pressure distribution across the sections $A-A$ and $A^{\prime}-A^{\prime}$ of the example in Section 9 (point load).

Pressure distributions of the problem obtained by both methods (9.1) and (9.2) are given in Fig. 7. Similar spurious pressure modes were obtained using method (9.1) when singular boundary conditions of primal variable are applied, such as the well-known driven-cavity problem which contains a rather severe singularity at the corner of the domain.

Similar experiments were run using the 4-node ( $Q_{1}$-elements) by 1-point integration. Interestingly enough, good results were obtained for smooth solutions on a uniform mesh and the method performs well in such cases. However, for distorted meshes and in the presence of irregular solutions, poor (oscillatory) pressures are again experienced.

\section{Added in Proof}

Olivier Jacquotte of TICOM has supplied us with a proof that the estimate $\alpha_{h}=O\left(h^{2}\right)$ in Theorem 6.3 can be improved to $\alpha_{h}=\mathrm{O}(h)$. The key is the construction of the sharper estimate: 


$$
\sum_{i=1}^{N}\left(\dot{q}_{i}^{1}-\stackrel{q}{q}_{i}^{3}\right)^{2}+\sum_{i=1}^{N}\left(\dot{q}_{i}^{2}-\stackrel{q}{q}_{i}^{4}\right)^{2} \geqslant C h^{2} \sum_{e=1}^{E} q_{e}^{2}
$$

(as opposed to the $\mathrm{O}\left(h^{4}\right)$ estimate given below (6.9)). The idea is to consider a case in which the origin of an integer coordinate system $(j, k)$ for node numbering is located at the node in the lower-left corner of the mesh, with the first node numbered $(1,1)$. We set $\dot{q}_{1}^{1}=\dot{q}_{2}^{2}=0$ and introduce a $C^{0}$-piecewise bilinear function $\phi$ which interpolates the $\stackrel{\circ}{q}_{e}$, for $j+k$ even at the nodes. One can show that

$$
\begin{aligned}
& \int_{\Omega^{\prime}}|\nabla \phi|^{2} \mathrm{~d} x \geqslant C_{1} \sum_{\substack{i=1 \\
j+k \text { even }}}^{N}\left[\left(\begin{array}{ll}
\dot{q}_{i}^{1} & \dot{q}_{i}^{3}
\end{array}\right)^{2}+\left(\dot{q}_{i}^{2}-\dot{q}_{i}^{4}\right)^{2}\right] \\
& \int_{\Omega^{\prime}}|\phi|^{2} \mathrm{~d} x \leqslant C_{2} h^{2} \sum_{\substack{e=1 \\
j+k \text { even }}}^{E} \stackrel{o}{q}_{e}^{2}
\end{aligned}
$$

where $\Omega^{\prime}$ is a rectangular domain centered $h / 2$ from $\partial \Omega$ inside $\Omega$. An application of Poincaré's inequality (which is possible because $\stackrel{q}{q}_{1}^{1}=\stackrel{q}{q}_{2}^{2}=0$ ) gives the desired result. Further details on this and related results are to be discussed in a forthcoming paper by Oden and Jacquotte.

\section{References}

[1] I. Babuska, The finite element method with Lagrange multipliers, Numer. Math. 20 (1973).

[2] I. Babuska and A.K. Aziz, Survey lecture on the mathematical foundations of the finite element method, in: The Mathematical Foundations of the Finite Element Method with Applications to Partial Differential Equations (Academic Press, New York, 1972).

[3] M. Bercovier, Perturbation of mixed variational problems-Applications to mixed finite element methods, R.A.I.R.O. 12(3) (1978) 211-236.

[4] M. Bercovier and M. Engelman, A finite element for the numerical solution of viscous incompressible flows, J. Comput. Phys., to appear.

[5] F. Brezzi, On the existence, uniqueness and approximation of saddle-point problems arising from Lagrangian multipliers, R.A.I.R.O. 8 (1974) 129-151.

[6] F. Brezzi, Sur l'existence, unicité, et approximation de problèmes de point de selle, C.R. Acad. Sci. Paris, Sér. A. 278 (1974) 839-842.

[7] P. Ciarlet, Numerical Analysis of the Finite Element Method for Elliptic Boundary Value Problems (North-Holland, Amsterdam, 1978).

[8] P. Ciarlet and P.A. Raviart, General Lagrange and Hermite interpolation in $\mathbb{R}^{n}$ with applications to finite element methods, Arch. Rational Mech. Anal. 46 (1972) 177-199.

[9] M. Crouzeix and P.A. Raviart, Conforming and nonconforming finite element methods for solving the stationary Stokes equations, R.A.I.R.O. 3 (1974) 33-76.

[10] M. Fortin, An analysis of the convergence of mixed finite element methods, R.A.I.R.O. 11(4) (1977) $341-354$.

[11] I. Fried, Finite element analysis of incompressible material by residual energy balancing. Internat. J. Solids and Structures 10 (1974) 993-1002.

[12] V. Girault and P.A. Raviart, An analysis of a mixed finite element method for the Navier-Stokes equations, Analyse Numérique et Fonctionelle, Université Paris VI, 1978.

[13] V. Girault and P.A. Raviart, Finite Element Approximation of the Navier-Stokes Equations, Lecture Notes in Mathematics 749 (Springer, New York, 1979).

[14] T.J.R. Hughes, Equivalence of finite elements for nearly incompressible elasticity, Tech. Rept. LBL 5237 , University of California, 1976.

[15] T.J.R. Hughes, Equivalence of finite elements for nearly incompressible elasticity, J. Appl. Mech. 44 (1977) $181-183$. 
[16] T.J.R. Hughes, R.L. Taylor and J.F. Levy, A finite element method for incompressible viscous flows, Second Internat. Symp. on Finite Element Methods in Flow Problems, S. Margherita Ligure, Italy, 1976.

[17] N. Kikuchi, Convergence of a penalty method for variational inequalities, TICOM Rept. 79-16, The University of Texas at Austin. Austin. TX, 1979.

[18] C. Johnson and J. Pitkaranta, Analysis of some mixed finite element methods related to reduced integration, Res. Rept., Dept. of Comput. Sci., Chalmers Univ. of Tech. and Univ. of Göteborg, 1980.

[19] O.A. Ladyszhenskaya, 'I he Mathematical Theory of Viscous Incompressible Flows (Gordon and Breach, New York, 1969).

[20] R.L. Lee, P.M. Gresho and R.L. Sani, Smoothing techniques for certain primitive variable solutions of the Navier-Stokes equations, Internat. J. Numer. Meths. Engrg. 14 (1979) 1785-1804.

[21] D.S. Malkus, Finite element analysis of incompressible solids, Dissertation, Dept. of Mathematics, Boston University, Boston, MA, 1972.

[22] J.N. Reddy, On the accuracy and existence of solutions to primitive variable models of viscous incompressible A unification of concepts, Comput. Meths. Appl. Mech. Engrg. 15 (1978) 63-81.

[23] J.N. Reddy, On the accuracy and existence of solutions to primitive variable models of viscous incompressible fluids, Internat. J. Engrg. Sci. 16 (1978) 921-929.

[24] R. Teman, Navier Stokes Equations (North-Holland, Amsterdam, 1977).

[25] O.C. Zienkiewicz, R.L. Taylor and J.M. Too, Reduced integration technique in general analysis of plates and shells, Internat. J. Numer. Meths. Engrg. 3 (1971) 275-290. 